TRANSACTIONS OF THE

AMERICAN MATHEMATICAL SOCIETY

Volume 353, Number 7, Pages 2805-2841

S 0002-9947(01)02722-2

Article electronically published on January 29, 2001

\title{
A MODEL STRUCTURE ON THE CATEGORY OF PRO-SIMPLICIAL SETS
}

\author{
DANIEL C. ISAKSEN
}

\begin{abstract}
We study the category pro- $\mathcal{S} \mathcal{S}$ of pro-simplicial sets, which arises in étale homotopy theory, shape theory, and pro-finite completion. We establish a model structure on pro-SS so that it is possible to do homotopy theory in this category. This model structure is closely related to the strict structure of Edwards and Hastings. In order to understand the notion of homotopy groups for pro-spaces we use local systems on pro-spaces. We also give several alternative descriptions of weak equivalences, including a cohomological characterization. We outline dual constructions for ind-spaces.
\end{abstract}

\section{INTRODUCTION}

If $\mathcal{C}$ is a category, then the pro-category pro- $\mathcal{C}$ [1 Exposé 1 , Section 8] is the category whose objects are small cofiltered systems in $\mathcal{C}$ (of arbitrary shape) and whose morphisms are given by the formula

$$
\operatorname{Hom}(X, Y)=\lim _{s} \operatorname{colim}_{t} \operatorname{Hom}_{\mathcal{C}}\left(X_{t}, Y_{s}\right) .
$$

While investigating the étale homotopy functor, Artin and Mazur 2] studied the category pro- $\mathrm{Ho}(\mathcal{S S})$, where $\mathrm{Ho}(\mathcal{S S})$ is the homotopy category of simplicial sets. They also introduced a notion of weak equivalence of pointed connected pro-spaces that involved isomorphisms of pro-homotopy groups.

However, an Artin-Mazur weak equivalence is not the same as an isomorphism in pro- $\mathrm{Ho}(\mathcal{S S})$. This suggests that pro- $\mathrm{Ho}(\mathcal{S S})$ is not quite the correct category for studying étale homotopy.

Around the same time, Quillen [14] developed the fundamental notions of homotopical algebra by realizing that model structures allow one to do homotopy theory in many different categorical contexts. A model structure on a category is a choice of three classes of maps (weak equivalences, cofibrations, and fibrations) satisfying certain axioms. The weak equivalences are inverted to obtain the associated homotopy category, while the cofibrations and fibrations serve an auxiliary role. Quillen 14. II.0.2] observed that the homotopy theory of pro-spaces would be an interesting application of model structures.

At least two model structures for pro-spaces are already known to exist. Edwards and Hastings [6] established a "strict" model structure on pro-spaces for the

Received by the editors October 12, 1999.

2000 Mathematics Subject Classification. Primary 18E35, 55Pxx, 55U35; Secondary 14F35, $55 \mathrm{P} 60$.

Key words and phrases. Closed model structures, pro-spaces, étale homotopy.

The author was supported in part by an NSF Graduate Fellowship.

(C)2001 American Mathematical Society 
purposes of shape theory and proper homotopy theory, but their weak equivalences did not generalize those of Artin and Mazur.

Also, Grossman [9] described a different model structure for pro-spaces that are countable towers. The weak equivalences of this theory are appropriately related to the Artin-Mazur equivalences. The category of towers is suitable in many applications from proper homotopy theory because it is reasonable to assume that the neighborhoods at infinity have a countable basis.

However, applications of pro-spaces to the algebro-geometric concept of étale cohomology require more general pro-spaces. General cofiltered systems of spaces are necessary for essentially the same reason that the sheaf theory of Grothendieck topologies is necessary to define étale cohomology.

This paper gives a generalization of Grossman's model structure to the entire category of pro-spaces. Weak equivalences between pointed connected pro-spaces are precisely Artin-Mazur weak equivalences. The Edwards-Hastings strict structure is an intermediate stage to building our structure. Our homotopy theory is the $P$-localization of the strict homotopy theory, where $P$ is the functor that replaces a space with its Postnikov tower.

Our weak equivalences have several alternative characterizations, one of which uses twisted cohomology. This is important because it is often difficult to check that a map of pro-spaces induces an isomorphism on homotopy groups. Usually it is much easier to verify a cohomology isomorphism and then conclude that the map is a weak equivalence.

Another characterization of weak equivalences is in terms of "eventually $n$ connected" maps (see Theorem $7.3(d)$ ), which is a very convenient property in practice. The equivalence of this property with the definition of weak equivalence is not obvious. One must use the full power of the model structure to prove the equivalence.

We mention two applications of this homotopy theory of pro-spaces. First, the model structure gives a more convenient category for studying étale homotopy because it allows the reinterpretation of the central ideas of the theory in terms of the established notions of model structures. It is also a start towards the definition of generalized cohomology of pro-spaces. For example, define $K^{0}$ to be represented by the constant pro-space $B U$. The realization of $K$-theory as a generalized cohomology theory requires more understanding of the category of pro-spectra. The étale $K$-theory of a scheme [5] is probably most clearly expressed as a generalized cohomology theory applied to the étale homotopy type.

Second, pro-spaces arise in the study of pro-finite completion [15] 44 13. Again, the new model structure provides a better category in which to study such completions. For example, Mandell [1] has used the model structure to compare his new algebraic construction of $p$-adic homotopy theory to Sullivan's $p$-pro-finite completion.

We describe briefly the model structure; the formal definitions appear in Section 6. Given a pointed pro-space $X$ (i.e., a map from the one-point constant pro-space * to $X$ or equivalently a pro-object in the category of pointed spaces), one can apply the functor $\pi_{n}(-, *)$ to each space in the pro-system to get a pro-group $\pi_{n}(X, *)$. The most obvious notion of equivalence of pro-spaces $X \rightarrow Y$ is the requirement that the map induce an isomorphism of pro-homotopy groups $\pi_{n}(X, *) \rightarrow \pi_{n}(Y, *)$ for every $n \geq 0$ and every point $*$ of $X$. 
However, points of a pro-space are rather awkward. In fact, some non-trivial prospaces have no points whatsoever. Local systems permit the discussion of homotopy groups without choosing basepoints. Grossman's work on towers 9] inspired this trick.

Cofibrations of pro-spaces are maps that are isomorphic to levelwise cofibrations of systems of spaces of the same shape. The model category axioms then force the definition of fibrations. These fibrations are similar to those of Edwards and Hastings [6, 3.3], but they satisfy an extra condition that compares the homotopy groups of the total pro-space to the homotopy groups of the base pro-space.

The model structure has a few interesting aspects. For example, it is not cofibrantly generated. This means that the proof of the model axioms is quite different from the standard arguments. One result of this fact is that the factorizations are not functorial. Almost all naturally arising model structures are cofibrantly generated. Hence pro-spaces are an interesting example of a non-cofibrantly generated model structure.

Also, we only know how to work with pro-simplicial sets, not pro-topological spaces. We use in a very significant way the fact that finite dimensional skeletons are functorial for simplicial sets. Since relative cell complexes do not have functorial skeletons, the same proofs do not apply.

Finally, note that the category of ind-spaces has a similar model structure. We do not provide details in this paper because we have no application in mind. Several comments throughout the paper explain where the significant differences occur.

The paper is divided into three main parts. Sections 25 give some background material and introduce language and tools for later use. Sections 6-10 describe the model structure, state the main theorems, and make comparisons to other homotopy theories. Sections 11119 provide proofs of the main theorems.

We assume familiarity with model structures. See [10] or [14] for background material.

Many of the important ideas in this paper come from Grossman [9]. I thank Peter May, Bill Dwyer, Brooke Shipley, Greg Arone, Michael Mandell, and Charles Rezk for many helpful conversations throughout the progress of this work.

\section{Preliminaries on Pro-Categories}

First we establish some terminology for pro-categories.

Definition 2.1. For a category $\mathcal{C}$, the category pro- $\mathcal{C}$ has objects all small cofiltering systems in $\mathcal{C}$, and

$$
\operatorname{Hom}_{\text {pro-e }}(X, Y)=\lim _{s} \operatorname{colim}_{t} \operatorname{Hom}_{\mathcal{C}}\left(X_{t}, Y_{s}\right) .
$$

Objects of pro- $\mathcal{C}$ can be thought of as functors from arbitrary small cofiltering categories to $\mathcal{C}$. See 1] or [2] for more background on the definition of pro-categories. We use both set theoretic and categorical language to discuss indexing categories; hence " $t \geq s$ " and " $t \rightarrow s$ " mean the same thing.

The word "system" always refers to an object of a pro-category, while the word "diagram" refers to a diagram of pro-objects.

A subsystem of an object $X: I \rightarrow \mathcal{C}$ in pro- $\mathrm{C}$ is a restriction of $X$ to a cofiltering subcategory $J$ of $I$. A subsystem is cofinal if for every $s$ in $I$, there exists some $t$ in $J$ and an arrow $t \rightarrow s$ in $I$. A system is isomorphic in pro- $\mathcal{C}$ to any of its cofinal subsystems. 
A directed set $I$ is cofinite if for every $t$, the set of elements of $I$ less than $t$ is finite. Except in Section 11, all systems are indexed by cofinite directed sets rather than arbitrary cofiltering categories. This is no loss of generality [, 2.1.6] (or [1, Exposé 1, 8.1.6]). The cofiniteness is critical because many constructions and proofs proceed inductively.

Whenever possible we avoid mentioning the structure maps of a pro-object $X$ explicitly. When necessary the notation $(X, \phi)$ indicates a system of objects $\left\{X_{s}\right\}$ with structure maps $\phi_{t s}: X_{t} \rightarrow X_{s}$.

Definition 2.2. Let $I$ be a cofinite directed set. For each $s$ in $I$, the height $h(s)$ of $s$ is the value of $n$ in the longest chain $s>s_{1}>s_{2}>\cdots>s_{n}$ starting at $s$ in $S$. In particular, $h(s)=0$ if and only if there are no elements of $I$ less than $s$.

We always assume that systems have no initial object or equivalently that any system has objects of arbitrarily large height. This is no loss of generality since it is possible to add isomorphisms to a system so that it no longer has an initial object, and this new system is isomorphic to the old one.

We frequently consider maps between two pro-objects with the same index categories. In this setting, a level map $X \rightarrow Y$ between pro-objects indexed by $I$ is given by maps $X_{s} \rightarrow Y_{s}$ for all $s$ in $I$. Up to isomorphism, every map is a level map [2, Appendix 3.2].

A map satisfies a certain property levelwise if it is a level map $X \rightarrow Y$ such that each $X_{s} \rightarrow Y_{s}$ satisfies that property.

Lemma 2.3. A level map $A \rightarrow B$ in pro-C is an isomorphism if and only if for all $s$, there exists $t \geq s$ and a commutative diagram

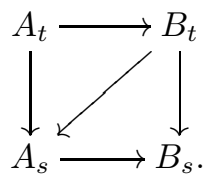

Proof. The maps $B_{t} \rightarrow A_{s}$ induce an inverse.

\section{Preliminaries on Simplicial Sets}

Now we review some definitions and results about simplicial sets. Let $\mathcal{S} \mathcal{S}$ be the category of simplicial sets. We use the expressions "space" and "simplicial set" interchangeably.

For simplification, we often use the same notation for a basepoint and its image under various maps (e.g., $\left.\pi_{n}(X, *) \rightarrow \pi_{n}(Y, *)\right)$.

Definition 3.1. A map $f: X \rightarrow Y$ of simplicial sets is an $n$-equivalence if for all basepoints $*$ in $X, f$ induces an isomorphism $\pi_{i}(X, *) \rightarrow \pi_{i}(Y, *)$ for $0 \leq i<n$ and a surjection $\pi_{n}(X, *) \rightarrow \pi_{n}(Y, *)$. The map $f$ is a co-n-equivalence if for all basepoints $*$ in $X, f$ induces an isomorphism $\pi_{i}(X, *) \rightarrow \pi_{i}(Y, *)$ for $i>n$ and an injection $\pi_{n}(X, *) \rightarrow \pi_{n}(Y, *)$.

Definition 3.2. Set

$$
J_{n}=\left\{\Lambda_{k}^{m} \rightarrow \Delta^{m} \mid m \geq 0\right\} \cup\left\{\partial \Delta^{m} \rightarrow \Delta^{m} \mid m>n\right\} .
$$

A map of simplicial sets is a co-n-fibration if it has the right lifting property with respect to all maps in $J_{n}$. A map of simplicial sets is an $n$-cofibration if it has the left lifting property with respect to all co- $n$-fibrations. 
In other words, a co- $n$-fibration is a $J_{n}$-injective, and an $n$-cofibration is a $J_{n^{-}}$ cofibration [10, 12.4.1]. Note that co- $n$-fibrations and $n$-cofibrations are characterized by lifting properties with respect to each other. When $n=\infty$ or $n=-1$, the definitions reduce to the usual definitions of trivial cofibrations and fibrations or to the definitions of cofibrations and trivial fibrations.

We will see below that $n$-cofibrations are just maps that are both cofibrations and $n$-equivalences. Also, co- $n$-fibrations are just maps that are both fibrations and co- $n$-equivalences. These facts motivate the terminology.

Proposition 3.3. For any $n$, each map $f$ of simplicial sets factors as $f=p i$, where $i$ is an $n$-cofibration and $p$ is a co-n-fibration.

Proof. Apply the small object argument [10, 12.4].

Lemma 3.4. A map $f: E \rightarrow B$ is a co-n-fibration if and only if $f$ is a fibration and co-n-equivalence.

Proof. First suppose that $f$ is a co- $n$-fibration. The generating trivial cofibrations are contained in $J_{n}$, so $f$ is also a fibration. Now we must show that $f$ is also a co- $n$-equivalence.

Consider test diagrams of the form

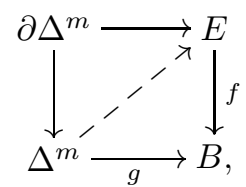

where $m>n$. Let $g: \Delta^{m} \rightarrow B$ be a constant map with image $*$, and let $F$ be the fiber of $f$ over $*$. Since lifts exist in the test diagram, $\pi_{m} F=0$ for $m \geq n$. Using the long exact sequence of homotopy groups of a fibration, it follows that $f$ is a co- $n$-equivalence.

Now suppose that $f$ is a fibration and co-n-equivalence. It follows from the long exact sequence of homotopy groups that $\pi_{m} F=0$ for $m \geq n$, where $F$ is any fiber of $f$. There are lifts in the test diagrams shown above for $m>n$ because the obstructions to such lifts belong to $\pi_{m-1} F$. Hence $f$ is a co- $n$-fibration.

Definition 3.5. If $A \rightarrow X$ is a cofibration of simplicial sets, then the relative $n$-skeleton $X^{(n)}$ is the union of $A$ and the $n$-skeleton of $X$.

Lemma 3.6. $A$ map $f: A \rightarrow X$ is an $n$-cofibration if and only if $f$ is a cofibration and $n$-equivalence.

Proof. Since trivial fibrations are co- $n$-fibrations, $n$-cofibrations are also cofibrations.

Suppose that $f: A \rightarrow X$ is a relative $J_{n}$-cell complex [10, 12.4.6]. Then $A \rightarrow$ $X^{(n)}$ is a weak equivalence since $X^{(n)}$ is obtained from $A$ by gluing along maps of the form $\Lambda_{k}^{m} \rightarrow \Delta^{m}$. Note that $X^{(n)} \rightarrow X$ is an $n$-equivalence, so $A \rightarrow X$ is also an $n$-equivalence. Arbitrary $n$-cofibrations are retracts of relative $J_{n}$-cell complexes [10, 13.2.9], so all $n$-cofibrations are $n$-equivalences.

Conversely, suppose that $f$ is a cofibration and $n$-equivalence. We show that $f$ is an $n$-cofibration by demonstrating that it has the left lifting property with respect to all maps that are both fibrations and co-n-equivalences. By Lemma 3.4, this means that $f$ has the left lifting property with respect to all co- $n$-fibrations. 
Factor $f$ as

$$
A \stackrel{j}{\longrightarrow} Y \stackrel{q}{\longrightarrow} X
$$

where $j$ is a trivial cofibration and $q$ is a fibration. Note that $q$ is also an $n$ equivalence. Let $p: E \rightarrow B$ be a map that is both a fibration and co- $n$-equivalence, and consider a diagram

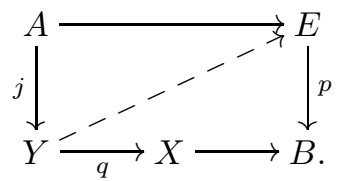

The dashed arrow exists because $j$ is a trivial cofibration and $p$ is a fibration.

This gives the diagram

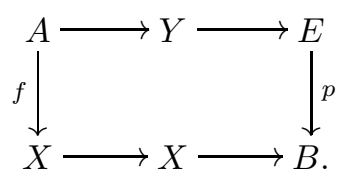

There is no obstruction to lifting over $X^{(0)}$ since $\pi_{0} A \rightarrow \pi_{0} X$ is surjective.

Obstructions to finding a lift over the higher relative skeletons of $X$ belong to $\pi_{m} F$, where $F$ is some fiber of $p$. These obstructions lie in the image of $\pi_{m} G$, where $G$ is a fiber of $q$. For every $m$, either $\pi_{m} G$ or $\pi_{m} F$ is zero. Hence there are no obstructions to lifting.

Remark 3.7. For ind-spaces, consider the set

$$
I_{n}=\left\{\Lambda_{k}^{m} \rightarrow \Delta^{m} \mid m \geq 0\right\} \cup\left\{\partial \Delta^{m} \rightarrow \Delta^{m} \mid m<n\right\}
$$

to define $n$-fibrations and co-n-cofibrations. All $n$-fibrations are both fibrations and $n$-equivalences, but the converse is not true. If $f: A \rightarrow X$ is a co- $n$-cofibration, then $f$ is a cofibration and the induced map $X^{(n-1)} \rightarrow X$ is a weak equivalence.

\section{LOCAL Systems}

The language of local systems is necessary in order to state the idea of homotopy groups for pro-spaces. Recall that a local system on a space $X$ is a functor $\Pi X \rightarrow$ $\mathrm{Ab}$, where $\Pi X$ is the fundamental groupoid of $X$ and $\mathrm{Ab}$ is the category of abelian groups. Denote by $\mathcal{L} \mathcal{S}(X)$ the category of local systems on $X$ or equivalently the category of locally constant sheaves on $X$.

For example, $\Pi_{n} X$ is a local system on $X$ for $n \geq 2$. It is defined by $\left(\Pi_{n} X\right)_{x}=$ $\pi_{n}(X, x)$ with isomorphisms $\left(\Pi_{n} X\right)_{x} \rightarrow\left(\Pi_{n} X\right)_{y}$ given by the usual maps on homotopy groups induced by paths.

Occasionally we refer to local systems with values in non-abelian groups. For example, $\Pi_{1} X$ is such a local system. We emphasize the notational distinction between $\Pi X$ (a groupoid) and $\Pi_{1} X$ (a local system).

If $f: X \rightarrow Y$ is a map of spaces, then $f$ induces a map of local systems $\Pi_{n} X \rightarrow$ $f^{*} \Pi_{n} Y$ on $X$, where $f^{*}$ is the pullback functor $\mathcal{L} \mathcal{S}(Y) \rightarrow \mathcal{L} \mathcal{S}(X)$. Recall that the functor $f^{*}$ is exact in the sense that it preserves finite limits and colimits, and it is also exact in the sense that it preserves exact sequences. 
Lemma 4.1. Let $f: X \rightarrow Y$ be a map of spaces. Then $f$ is a weak equivalence if and only if $\pi_{0} f$ is an isomorphism and $\Pi_{n} X \rightarrow f^{*} \Pi_{n} Y$ is an isomorphism of local systems on $X$ for all $n \geq 1$.

Proof. This is a restatement without reference to basepoints of the definition of weak equivalence of simplicial sets.

We now extend the definitions to pro-spaces.

Definition 4.2. A local system on a pro-space $X$ is an object of $\operatorname{colim}_{s} \mathcal{L} \mathcal{S}\left(X_{s}\right)$. If $L$ is a local system on $(X, \phi)$ represented by a functor $L_{s}: \Pi X_{s} \rightarrow \mathrm{Ab}$ and $M$ is another local system on $X$ represented by a functor $M_{t}: \Pi X_{t} \rightarrow$ Ab, then a map from $L$ to $M$ is an element of $\operatorname{colim}_{u} \operatorname{Hom}_{\mathcal{L} s\left(X_{u}\right)}\left(\phi_{u s}^{*} L_{s}, \phi_{u t}^{*} M_{t}\right)$. Denote by $\mathcal{L} \mathcal{S}(X)$ the category of local systems on $X$.

A local system on $X$ is represented by a local system on $X_{s}$ for some $s$. For example, for $n \geq 1, \Pi_{n} X_{s}$ is a local system on $X$ for each $s$.

A map between two local systems on $X$ is a map of representing local systems pulled back to some $X_{u}$. For example, for $n \geq 1$ and $t \geq s, \Pi_{n} X_{t} \rightarrow \Pi_{n} X_{s}$ is a map of local systems on $(X, \phi)$ because $\Pi_{n} X_{t} \rightarrow \phi_{t s}^{*} \Pi_{n} X_{s}$ is a map of local systems on $X_{t}$.

Let $f: X \rightarrow Y$ be a map of pro-spaces, and let $L: \Pi Y_{s} \rightarrow$ Ab be a local system on $Y_{s}$. Choose any map $f_{t s}: X_{t} \rightarrow Y_{s}$ representing $f$ and consider the functor $L \circ f_{t s}: \Pi X_{t} \rightarrow \Pi Y_{s} \rightarrow \mathrm{Ab}$. This gives a well-defined functor $\operatorname{colim}_{s} \mathcal{L} \mathcal{S}\left(Y_{s}\right) \rightarrow$ $\operatorname{colim}_{t} \mathcal{L} \mathcal{S}\left(X_{t}\right)$.

Definition 4.3. Let $f: X \rightarrow Y$ be a map of pro-spaces. The pullback $f^{*}$ : $\mathcal{L S}(Y) \rightarrow \mathcal{L S}(X)$ is the functor $\operatorname{colim}_{s} \mathcal{L S}\left(Y_{s}\right) \rightarrow \operatorname{colim}_{t} \mathcal{L S}\left(X_{t}\right)$.

Lemma 4.4. If $f: X \rightarrow Y$ is a map of pro-spaces, then $f^{*}: \mathcal{L} \mathcal{S}(Y) \rightarrow \mathcal{L} \mathcal{S}(X)$ is an exact functor in the sense that it preserves finite limits and colimits.

Proof. Without loss of generality, we may assume that $f$ is a level map. Given a finite diagram of local systems $L$ on $Y$, there is an $s$ such that each $L^{i}$ is represented by a local system $L_{s}^{i}$ on $Y_{s}$. Then for each $i, f^{*} L^{i}$ is represented by $f_{s}^{*} L_{s}^{i}$. Now colim $L^{i}$ in $\mathcal{L S}(Y)$ is represented by colim $L_{s}^{i}$ in $\mathcal{L} \mathcal{S}\left(Y_{s}\right)$, so $f^{*}\left(\operatorname{colim} L^{i}\right)$ is represented by $f_{s}^{*}\left(\operatorname{colim} L_{s}^{i}\right)$. Also, $\operatorname{colim} f^{*} L^{i}$ in $\mathcal{L} \mathcal{S}(X)$ is represented by colim $f_{s}^{*} L_{s}^{i}$ in $\mathcal{L S}\left(X_{s}\right)$. But $f_{s}^{*}$ commutes with finite colimits, so colim $f^{*} L^{i}$ and $f^{*}\left(\operatorname{colim} L^{i}\right)$ are isomorphic since they are represented by the same local system on $X_{s}$.

An identical argument shows that $f^{*}$ commutes with finite limits.

It follows from the lemma that $f^{*}$ is exact in the sense that it preserves exact sequences.

\section{5. Номотору GRoups}

With the notions of local systems in place, we can define homotopy groups of pro-spaces as pro-objects in a category of local systems. The local systems are necessary to avoid mentioning basepoints.

Definition 5.1. If $X$ is a pro-space and $n \geq 2$, then $\Pi_{n} X$ is the pro-local system on $X$ given by $\left\{\Pi_{n} X_{s}\right\}$. Also, $\pi_{0} X$ is the pro-set given by $\left\{\pi_{0} X_{s}\right\}$, and $\Pi_{1} X$ is the pro-local system on $X$ with values in non-abelian groups given by $\left\{\Pi_{1} X_{s}\right\}$. 
Note that a map of pro-spaces $f: X \rightarrow Y$ induces a map $\Pi_{n} X \rightarrow f^{*} \Pi_{n} Y$ in $\operatorname{pro}-\mathcal{L} \mathcal{S}(X)$.

Lemma 5.2. If $f: X \rightarrow Y$ is a map of pro-spaces and $\pi_{0} f$ is an epimorphism in the category of pro-sets, then a map $g$ of pro-local systems is an isomorphism in pro- $\mathcal{L} \mathcal{S}(Y)$ if and only if $f^{*}(g)$ is an isomorphism in pro- $\mathcal{L} \mathcal{S}(X)$.

Proof. Without loss of generality, assume that $f$ is a level map. Note that pro- $\mathcal{L} \mathcal{S}(Y)$ is an abelian category [2, Appendix 4.5]. Since $f^{*}$ is exact, it suffices to consider a local system $L$ on $Y$ such that $f^{*} L=0$ and conclude that $L=0$.

A pro-local system $M$ is zero if and only if for every $i$, there exists $j \geq i$ such that the map $M^{j} \rightarrow M^{i}$ is trivial. For any $i$, choose $j \geq i$ so that $f^{*} L^{j} \rightarrow f^{*} L^{i}$ is trivial. Let $L_{s}^{i}$ and $L_{s}^{j}$ be local systems on $Y_{s}$ representing $L^{i}$ and $L^{j}$ respectively. Choose $s$ large enough so that $f_{s}^{*} L_{s}^{j} \rightarrow f_{s}^{*} L_{s}^{i}$ is a trivial map of local systems on $X_{s}$.

Since $\pi_{0} f$ is an epimorphism, there exists some $t$ and a map $\pi_{0} Y_{t} \rightarrow \pi_{0} X_{s}$ such that the map $\pi_{0} Y_{t} \rightarrow \pi_{0} Y_{s}$ factors through $\pi_{0} X_{s}$. Since $f_{s}^{*} L_{s}^{j} \rightarrow f_{s}^{*} L_{s}^{i}$ is trivial, the map $L_{s}^{j} \rightarrow L_{s}^{i}$ is trivial when restricted to the components of $Y_{s}$ in the image of $X_{s}$. Now the image of $\pi_{0} X_{s}$ in $\pi_{0} Y_{s}$ contains the image of $\pi_{0} Y_{t}$, so $L_{s}^{j} \rightarrow L_{s}^{i}$ becomes trivial when pulled back to $Y_{t}$. Hence the map $L^{j} \rightarrow L^{i}$ is trivial. This means that the pro-local system $L$ is zero.

Remark 5.3. A similar statement is true for pro-local systems with values in nonabelian groups.

Lemma 5.4. A map of pro-spaces $f: X \rightarrow Y$ induces an isomorphism of pro-sets $\pi_{0} f: \pi_{0} X \rightarrow \pi_{0} Y$ if and only if $f$ is isomorphic to a level map $f^{\prime}: X^{\prime} \rightarrow Y^{\prime}$ such that $f^{\prime}$ induces a level isomorphism $\pi_{0} f^{\prime}: \pi_{0} X^{\prime} \rightarrow \pi_{0} Y^{\prime}$.

Proof. One direction is clear because level isomorphisms are pro-isomorphisms. Assume that $\pi_{0} f$ is an isomorphism. We may also assume that $f$ is a level map.

Define $X^{\prime}=X$ and $Y^{\prime}=Y \times_{\pi_{0} Y} \pi_{0} X$. Here we identify pro-sets with pro-spaces of dimension zero.

Then $Y^{\prime}$ is isomorphic to $Y$ since $\pi_{0} X \rightarrow \pi_{0} Y$ is an isomorphism. Let $f^{\prime}: X^{\prime} \rightarrow$ $Y^{\prime}$ be the map induced by $f: X \rightarrow Y$ and the projection $X \rightarrow \pi_{0} X$. Pullbacks can be constructed levelwise in pro-categories, so for all $s, Y_{s}^{\prime}=Y_{s} \times_{\pi_{0} Y_{s}} \pi_{0} X_{s}$ and $f_{s}^{\prime}$ is induced by $f_{s}: X_{s} \rightarrow Y_{s}$ and $X_{s} \rightarrow \pi_{0} X_{s}$. Note that $f_{s}^{\prime}$ induces an isomorphism $\pi_{0} X_{s}^{\prime} \rightarrow \pi_{0} Y_{s}^{\prime}$. Hence $f^{\prime}$ is the desired map.

If $f: X \rightarrow Y$ is a map of spaces such that $\pi_{0} f$ is an isomorphism and $\pi_{1} f$ is an isomorphism for every basepoint, then $f^{*}$ induces an equivalence of categories $\mathcal{L S}(Y) \rightarrow \mathcal{L S}(X)$. The following lemma makes an analogous statement for prospaces.

Lemma 5.5. If $f: X \rightarrow Y$ is a map of pro-spaces such that $\pi_{0} f$ and $\Pi_{1} X \rightarrow$ $f^{*} \Pi_{1} Y$ are isomorphisms, then the functor $f^{*}: \mathcal{L S}(Y) \rightarrow \mathcal{L} \mathcal{S}(X)$ is an equivalence of categories.

Proof. We only prove that $f^{*}$ is essentially surjective in the sense that every object $L$ of $\mathcal{L} \mathcal{S}(X)$ is isomorphic to $f^{*} M$ for some $M$ in $\mathcal{L S}(Y)$. We leave the rest of the proof to the interested reader. We will use only the surjectivity in this work.

With no loss of generality, we may assume that $f$ is a level map. By Lemma 5.4 we may also assume that $\pi_{0} f$ is a level isomorphism. 
Let $L$ be a local system on $(X, \phi)$ represented by a local system $L_{s}$ on $X_{s}$. There exists $t \geq s$ and a commutative diagram

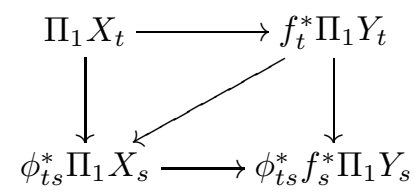

of local systems on $X_{t}$.

Choose one point $x_{i}$ in each component of $X_{t}$. Let $y_{i}$ be the image of $x_{i}$ in $Y_{t}$; this is a choice of one point in each component of $Y_{t}$. Let $x_{i}^{\prime}$ be the image of $x_{i}$ in $X_{s}$. as

By evaluating the above diagram at $x_{i}$, the map $\pi_{1}\left(X_{t}, x_{i}\right) \rightarrow \pi_{1}\left(X_{s}, x_{i}^{\prime}\right)$ factors

$$
\pi_{1}\left(X_{t}, x_{i}\right) \longrightarrow \pi_{1}\left(Y_{t}, y_{i}\right) \stackrel{g_{i}}{\longrightarrow} \pi_{1}\left(X_{s}, x_{i}^{\prime}\right) .
$$

The maps $g_{i}: \pi_{1}\left(Y_{t}, y_{i}\right) \rightarrow \pi_{1}\left(X_{s}, x_{i}^{\prime}\right)$ and the local system $L_{s}$ determine a local system $M_{t}$ on $Y_{t}$ by setting $\left(M_{t}\right)_{y_{i}}=L_{x_{i}^{\prime}}$ with the $\pi_{1}\left(Y_{t}, y_{i}\right)$-action induced by $g_{i}$.

Let $M$ be the local system on $Y$ represented by $M_{t}$. Note that $f_{t}^{*} M_{t}$ is isomorphic to $\phi_{t s}^{*} L_{s}$. Hence $f^{*} M$ is isomorphic to $L$.

Remark 5.6. A similar statement applies to local systems with values in non-abelian groups.

\section{Model Structure}

Now we explicitly describe the model structure on the category of pro-spaces.

Definition 6.1. A map of pro-spaces $f: X \rightarrow Y$ is a weak equivalence if $\pi_{0} f$ is an isomorphism of pro-sets and $\Pi_{n} X \rightarrow f^{*} \Pi_{n} Y$ is an isomorphism in pro- $\mathcal{L} \mathcal{S}(X)$ for all $n \geq 1$.

In Corollary [7.5 we will see that for pointed connected pro-spaces, a level map $X \rightarrow Y$ is a weak equivalence if and only if $\pi_{n} X \rightarrow \pi_{n} Y$ is a pro-isomorphism for all $n$. This works because there is no need for arbitrary basepoints. Hence Artin-Mazur weak equivalences [2, Section 4] are also weak equivalences.

Definition 6.2. A map of pro-spaces is a cofibration if it is isomorphic to a levelwise cofibration.

Definition 6.3. A map of pro-spaces is a fibration if it has the right lifting property with respect to all trivial cofibrations.

Theorem 6.4. The above definitions give a proper simplicial model structure on pro-SS (without functorial factorizations). This model structure is not cofibrantly generated.

Proof. The axioms for a proper simplicial model structure are verified in Sections 11] through [17]

Limits and colimits exist by Proposition 11.1 The two-out-of-three axiom is Proposition 13.1. Retracts preserve weak equivalences because weak equivalences are defined in terms of isomorphisms. Retracts preserve fibrations because retracts preserve lifting properties. Corollary 12.2 is the retract axiom for cofibrations. Propositions 15.1 and 15.2 are the factoring axioms, while Proposition 15.4 is the 
non-trivial lifting axiom. The axioms for a simplicial model structure are demonstrated in Proposition [16.3 Proposition 17.1 shows that the model structure is proper, and Corollary 19.3) states that it is not cofibrantly generated.

The model structure can be considered in two stages. The "strict" structure of Edwards and Hastings [6, 3.3], in which the weak equivalences are defined levelwise, is an intermediate step; see Section 10 for details. The situation is not unlike the Bousfield-Friedlander strict and stable model structures for spectra [3].

We assume Theorem 6.4 for the rest of this section and for the next three sections. Sections 11-19 contain the details of the proof of the theorem.

In practice we need a more concrete description of fibrations. The next definition and proposition provide such a description.

Definition 6.5. A map is a strong fibration if it is isomorphic to a level map of pro-spaces $X \rightarrow Y$ indexed by a cofinite directed set such that for all $t$,

$$
X_{t} \rightarrow Y_{t} \times \lim _{s<t} Y_{s} \lim _{s<t} X_{s}
$$

is a co- $n(t)$-fibration, where $n$ is any function from the index set of $X$ to $\mathbb{N}$.

Proposition 14.2 shows that we could have taken the equivalent definition that $X_{t} \rightarrow Y_{t} \times_{\lim _{s<t} Y_{s}} \lim _{s<t} X_{s}$ is a fibration and $X_{t} \rightarrow Y_{t}$ is a co- $n(t)$-equivalence. Formally this alternative definition is more awkward, but it is often useful to recognize that specific examples are strong fibrations.

Note that this definition only applies to level maps indexed by cofinite directed sets. In fact, the obvious generalization to systems of arbitrary shape is incorrect. This suggests that the above definition has a more natural equivalent formulation, but we do not know how to restate it.

With no loss of generality, we always assume that $n(t) \geq n(s)$ whenever $t \geq s$.

It is not obvious from the definition that strong fibrations are also fibrations. The technical heart of the whole theory lies in showing that strong fibrations have the right lifting property with respect to trivial cofibrations.

Proposition 6.6. A map $f: E \rightarrow B$ of pro-spaces is a fibration if and only if there exists a strong fibration $q: E^{\prime} \rightarrow B$ and a commutative diagram

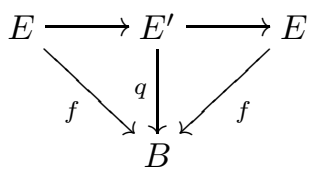

such that the composition along the top row is the identity on $E$.

Remark 6.7. The idea is that $f$ is a retract of $q$ in a strong sense where the targets of $f$ and $q$ are the same.

Proof. The "if" direction follows from the fact that strong fibrations are fibrations by Proposition 14.5 and the fact that retracts preserve fibrations.

Conversely, suppose that $f$ is a fibration. Use Proposition 15.1 to factor $f$ as

$$
E \stackrel{j}{\longrightarrow} E^{\prime} \stackrel{q}{\longrightarrow} B,
$$

where $j$ is a trivial cofibration and $q$ is a strong fibration. The retract argument [10, 8.2.2] finishes the proof. 
Proposition 6.8. A level map $f: A \rightarrow X$ of pro-spaces is a trivial cofibration if and only if there exists a levelwise cofibration $i: A \rightarrow X^{\prime}$ indexed by a cofinite directed set $I$ for which there is a strictly increasing function $n: I \rightarrow \mathbb{N}$ such that $i_{s}$ is an $n(s)$-equivalence and there is a commutative diagram

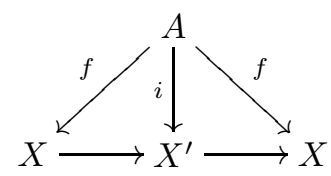

in which the composition along the bottom row is the identity on $X$.

Remark 6.9. The idea is that $f$ is a retract of $i$ in a strong sense where the sources of $f$ and $i$ are the same.

Proof. Suppose that $i$ satisfies the conditions of the proposition. Then $i$ is a cofibration by assumption, and it is a weak equivalence by Corollary 13.5. Therefore, $f$ is a retract of a trivial cofibration, so it is also a trivial cofibration.

Now suppose that $f$ is a trivial cofibration. Use Proposition 15.1 to factor $f$ as

$$
A \stackrel{i}{\longrightarrow} X^{\prime} \stackrel{q}{\longrightarrow} X,
$$

where $i$ satisfies the conditions of the proposition and $q$ is a strong fibration. By Proposition 14.5, $f$ has the left lifting property with respect to $q$. The retract argument [10, 8.2.2] finishes the proof.

Remark 6.10. Weak equivalences of ind-spaces are slightly more difficult to define because the language of local systems does not work so well. See Remark 7.4 for a rough description of weak equivalences of ind-spaces. The model structure for ind-spaces is again a localization of the "strict" structure of Edwards and Hastings [6, 3.8], so levelwise weak equivalences are weak equivalences in our sense.

Fibrations of ind-spaces are levelwise fibrations. Cofibrations are defined by the appropriate left lifting property. A dual notion of strong cofibration is important in this context. The technical heart of the theory lies in showing that strong cofibrations have the left lifting property with respect to trivial fibrations.

\section{Whitehead Theorem}

Definition 6.1 is useful for studying the model structure, but there are other characterizations of weak equivalences. We need a few preliminary definitions.

Definition 7.1. Let $X$ be a pro-space and let $L$ be a local system on $(X, \phi)$ represented by a functor $L_{s}: \Pi X_{s} \rightarrow \mathrm{Ab}$. Define the twisted cohomology groups

$$
H^{i}(X ; L)=\operatorname{colim}_{t \geq s} H^{i}\left(X_{t} ; \phi_{t s}^{*} L_{s}\right) .
$$

If $f: Y \rightarrow X$ is a level cofibration, then

$$
H^{i}(X, Y ; L)=\operatorname{colim}_{t \geq s} H^{i}\left(X_{t}, Y_{t} ; \phi_{t s}^{*} L_{s}\right)
$$

Note that there is a long exact sequence

$$
\cdots \longrightarrow H^{i}(X, Y ; L) \longrightarrow H^{i}(X ; L) \longrightarrow H^{i}\left(Y ; f^{*} L\right) \longrightarrow H^{i+1}(X, Y ; L) \longrightarrow \cdots
$$

since filtered colimits are exact. 
Recall that the Moore-Postnikov system [12, 8.9] of a space $X$ is a tower

$$
X \rightarrow \cdots \rightarrow P_{n} X \rightarrow P_{n-1} \rightarrow \cdots P_{1} X \rightarrow P_{0} X
$$

such that $X \cong \lim _{n} P_{n} X$, each map $P_{n} X \rightarrow P_{n-1} X$ is a fibration, and each space $P_{n} X$ has the same homotopy groups as $X$ in dimensions less than or equal to $n$ but has trivial homotopy groups in dimensions greater than $n$.

Definition 7.2. If the functor $X: I \rightarrow \mathcal{S} \mathcal{S}$ is a pro-space, then $P X$ is the pro-space given by the functor $\mathbb{N} \times I \rightarrow \mathcal{S} \mathcal{S}$ which sends $(n, s)$ to $P_{n} X_{s}$.

Note that Artin and Mazur [2] used a similar construction $X^{\natural}$ instead of $P X$.

Theorem 7.3. Suppose that $f: X \rightarrow Y$ is a map of pro-spaces. The following conditions are equivalent:

(a) $f$ is a weak equivalence.

(b) $f$ is isomorphic to a level map of pro-spaces $g: Z \rightarrow W$ such that for all $n \geq 0$ and for all $s$, there exists some $t \geq s$ such that for all basepoints $*$ in $Z_{t}$, there is a commutative diagram

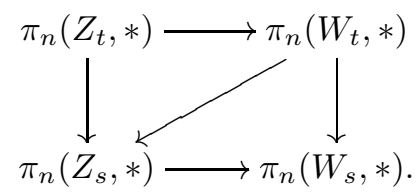

(c) $\pi_{0}(f)$ is an isomorphism of pro-sets, $\Pi_{1} X \rightarrow f^{*} \Pi_{1} Y$ is an isomorphism of pro-local systems on $X$, and for all $i$ and all local systems $L$ on $Y$, the map $H^{i}(Y ; L) \rightarrow H^{i}\left(X ; f^{*} L\right)$ is an isomorphism.

(d) $f$ is isomorphic to a level map $g: Z \rightarrow W$ indexed by a cofinite directed set $I$ for which there is a strictly increasing function $n: I \rightarrow \mathbb{N}$ such that $g_{s}: Z_{s} \rightarrow W_{s}$ is an $n(s)$-equivalence.

(e) $P f$ is a strict weak equivalence.

We give the proof in Section 18

Condition (b) is a less elegant but more technically convenient definition of weak equivalences. It is precisely Grossman's definition [9] of weak equivalences for towers. Condition (c) generalizes the well-known result about spaces that a weak equivalence between simply connected spaces is the same as a cohomology isomorphism. Condition (d) gives the simplest description of weak equivalences. This condition is usually easiest to use when working with the homotopy theory of prospaces. Condition (e) says that our homotopy theory is the "P-localization" of the strict homotopy theory. See Section 10 for the definition of strict weak equivalences.

The proof that condition $(d)$ is equivalent to $(a)$ relies heavily on the model structure. Condition (d) could have been the definition of weak equivalences. This would make the proofs of the model structure axioms easier, but then it would be difficult to establish a link between such weak equivalences and pro-homotopy groups or cohomology groups.

Remark 7.4. We take the dual to condition (b) as the definition of weak equivalences of ind-spaces. This definition is equivalent to a dual version of condition $(d)$, but we do not know whether duals to conditions (a), (c), and (e) exist.

If $X$ is a pointed connected pro-space, then $\pi_{n} X$ is the pro-group given by the diagram $\left\{\pi_{n} X_{s}\right\}$. These are the pro-homotopy groups of Artin and Mazur [2]. The 
following corollary indicates that our weak equivalences appropriately generalize those of Artin and Mazur.

Corollary 7.5. If $f: X \rightarrow Y$ is a map of connected pointed pro-spaces, then $f$ is a weak equivalence if and only if $\pi_{n} f: \pi_{n} X \rightarrow \pi_{n} Y$ is an isomorphism of pro-groups for all $n$.

Proof. We may assume that $f$ is a level map. Suppose that the diagrams of condition (b) of Theorem 7.3 exist. Using Lemma 2.3, it follows that $\pi_{n} f: \pi_{n} X \rightarrow \pi_{n} Y$ is an isomorphism of pro-groups.

Now suppose that $\pi_{n} f: \pi_{n} X \rightarrow \pi_{n} Y$ is an isomorphism of pro-groups. Then the diagrams of Theorem 7.3 (b) exist for every $s$ when $*$ is the chosen basepoint of $X$.

Let $\sharp$ be any point of $X_{t}$. Choose a path from $\sharp$ to $*$ in $X_{t}$. This path induces an isomorphism between the diagram of Theorem $7.3(b)$ based at $*$ and the diagram based at $\sharp$.

\section{Comparison with Other Theories}

This section explains the difference between our homotopy category of pro-spaces and the homotopy category of Artin and Mazur [2]. We use the notations

$$
\begin{aligned}
{[K, L] } & =\operatorname{Hom}_{\mathrm{Ho}(\delta s)}(K, L), \\
{[X, Y]_{\text {pro }} } & =\operatorname{Hom}_{\mathrm{Ho}(\text { pro-ss })}(X, Y) .
\end{aligned}
$$

Let $c: S \mathcal{S} \rightarrow$ pro-SS be the functor given by constant pro-spaces.

Artin and Mazur [2] defined a homotopy category of pro-spaces by considering morphisms in $\lim _{t} \operatorname{colim}_{s}\left[X_{s}, Y_{t}\right]$. Problems with this category arise from the fact that diagrams commute only up to homotopy. More importantly, isomorphisms in their homotopy category are not the same as maps that induce isomorphisms of pro-homotopy groups.

If $X$ is cofibrant and $Y$ is fibrant, then, in general, $[X, Y]_{\text {pro }}$ is not equal to $\lim _{t} \operatorname{colim}_{s}\left[X_{s}, Y_{t}\right]$. In the pointed case, there is a spectral sequence involving higher $\lim ^{i}$ terms relating the two sets. In some special cases there is a simpler relationship.

Lemma 8.1. Let $X$ be a pro-space and let $K$ be a simplicial set with finitely many nonzero homotopy groups. Then

$$
[X, c K]_{\text {pro }} \cong \operatorname{colim}_{s}\left[X_{s}, K\right] .
$$

Proof. Let $K^{\prime}$ be a fibrant approximation of $K$. Then $c K^{\prime}$ is a fibrant approximation of $c K$ in the category of pro-spaces. Hence $[X, c K]_{\text {pro }}$ equals $\left[X, c K^{\prime}\right]_{\text {pro }}$ and $\operatorname{colim}_{s}\left[X_{s}, K\right]$ equals colims ${ }_{s}\left[X_{s}, K^{\prime}\right]$. Therefore, it suffices to assume that $K$ is fibrant.

According to Definition 16.2 $\operatorname{Map}(X, c K)=\operatorname{colim}_{s} \operatorname{Map}\left(X_{s}, K\right)$. Since $\pi_{0}$ commutes with filtered colimits,

$$
\pi_{0} \operatorname{Map}(X, c K) \cong \operatorname{colim}_{s} \pi_{0} \operatorname{Map}\left(X_{s}, K\right) .
$$

Since $X$ is cofibrant and $c K$ is fibrant, $[X, c K]_{\text {pro }}$ is equal to $\pi_{0} \operatorname{Map}(X, c K)$, and $\left[X_{s}, K\right]$ is equal to $\pi_{0} \operatorname{Map}\left(X_{s}, K\right)$ for every $s$. Therefore,

$$
[X, c K]_{\mathrm{pro}} \cong \operatorname{colim}_{s}\left[X_{s}, K\right] \text {. }
$$


For example, if $K=K(\pi, n)$, then

$$
[X, K]_{\mathrm{pro}} \cong \operatorname{colim}_{s}\left[X_{s}, K\right] \cong \operatorname{colim}_{s} H^{n}(X ; \pi) \cong H^{n}(X ; \pi) .
$$

This explains why the ideas of Artin and Mazur were good enough for studying ordinary cohomology. However, other theories such as $K$-theory present complications because they are not represented by spaces with only finitely many nonzero homotopy groups. Therefore, Artin and Mazur were not able to define $K$-theory for pro-spaces. We propose that $K$-theory be defined as the functor represented by $B U$. In order to calculate $K$-theory, one must use the Postnikov system $P B U$ of $B U$. This tower is a fibrant replacement for $B U$. For a general pointed pro-space $X$, there is a short exact sequence

$$
0 \rightarrow \lim _{n}^{1} \operatorname{colim}_{s}\left[\Sigma X_{s}, P_{n} B U\right] \rightarrow[X, B U]_{\text {pro }} \rightarrow \lim _{n} \operatorname{colim}_{s}\left[X_{s}, P_{n} B U\right] \rightarrow 0 .
$$

When $X$ is the étale homotopy type of a scheme $S$ over a separably closed field, the third term in this sequence is Friedlander's definition [7] of the étale $K$-theory of $S$. Hence our definition differs by a $\lim ^{1}$ error term.

The theory of generalized cohomology for pro-spaces should clarify the definition of the étale $K$-theory of schemes. Most likely the constructions of Dwyer and Friedlander [5] can be put into this framework for a cleaner description of the ideas. We plan to elaborate on this relationship in future work.

Proposition 8.2. The functors $c: \mathcal{S} \mathcal{S} \rightarrow$ pro-SS and $\mathrm{lim}:$ pro-SS $\rightarrow$ SS are a Quillen adjoint pair.

Proof. Let $X$ be a space and let $Y$ be a pro-space. Then

$$
\operatorname{Hom}_{\mathcal{S} S}\left(X, \lim _{s} Y_{s}\right)=\lim _{s} \operatorname{Hom}_{\mathcal{S} S}\left(X, Y_{s}\right)=\operatorname{Hom}_{\text {pro-ss }}(c X, Y) .
$$

Therefore $c$ and lim are an adjoint pair.

Since $c$ preserves cofibrations and trivial cofibrations, the functors are a Quillen pair.

Corollary 8.3. The functors $c$ and lim induce adjoint functors

$$
\mathrm{Ho}(\mathcal{H S}) \rightarrow \mathrm{Ho}(\text { pro-SS })
$$

and $\mathrm{Ho}($ pro-SS $) \rightarrow \mathrm{Ho}(\mathcal{S S})$.

The following proposition is a kind of dual to Lemma 8.1.

Proposition 8.4. Let $K$ be a simplicial set, and let $Y$ be a pro-space. Then

$$
[c K, Y]_{\text {pro }}=\left[K, \underset{s}{\operatorname{holim}} Y_{s}\right] .
$$

Proof. From Corollary 8.3 , we know that $[c K, Y]_{\text {pro }}$ is equal to $\left[K, \lim _{t} Z_{t}\right]$, where $Z$ is a fibrant approximation of $Y$. It suffices to show that $\lim _{t} Z_{t}$ is weakly equivalent to $\operatorname{holim}_{s} Y_{s}$.

For each $s$, let $Y_{s}^{\prime}$ be a functorial fibrant approximation of $Y_{s}$. Then $Y \rightarrow Y^{\prime}$ is a strict weak equivalence. Now let $Z$ be a strictly fibrant approximation of $P Y^{\prime}$. Note that $Z$ is a fibrant approximation of $Y$.

Recall that $Y_{s}^{\prime}$ is isomorphic to $\lim _{n} P_{n} Y_{s}^{\prime}$ for each $s$. Also, $Y_{s}^{\prime}$ is weakly equivalent to $\operatorname{holim}_{n} P_{n} Y_{s}^{\prime}$ since each map $P_{n} Y_{s}^{\prime} \rightarrow P_{n-1} Y_{s}^{\prime}$ is a fibration.

Now $\operatorname{holim}_{s} Y_{s}$ is weakly equivalent to $\operatorname{holim}_{s} Y_{s}^{\prime}$ since $Y$ and $Y^{\prime}$ are levelwise weakly equivalent. Further, $\operatorname{holim}_{s} Y_{s}^{\prime}$ is weakly equivalent to $\operatorname{holim}_{s} \operatorname{holim}_{n} P_{n} Y_{s}^{\prime}$. In other words, $\operatorname{holim}_{s} Y_{s}$ is weakly equivalent to $\operatorname{holim}_{n, s} P_{n} Y_{s}^{\prime}$. Since $Z$ is a strictly 
fibrant approximation of $P Y^{\prime}, \lim _{t} Z_{t}$ is weakly equivalent to $\operatorname{holim}_{n, s} P_{n} Y_{s}^{\prime}$ [6] $4.2]$.

Edwards and Hastings [6, 4.2] showed that homotopy limit is the derived functor of limit with respect to the strict structure. The proposition makes the stronger claim that homotopy limit is the derived functor of limit with respect also to our model structure. In other words, pro-spaces with isomorphic pro-homotopy groups have weakly equivalent homotopy limits, even if they are not levelwise weakly equivalent.

The proposition implies that the pro- $K$-theory described above restricts to ordinary topological $K$-theory on constant pro-spaces because $B U$ is weakly equivalent to $\operatorname{holim}_{n} P_{n} B U$.

\section{Pro-Finite Completion}

Now we make a comparison between the homotopy category of pro-spaces and Morel's homologically defined homotopy theory on pro-simplicial finite sets [13.

Let $\mathcal{S} \mathcal{F} \mathcal{S}$ be the category of simplicial finite sets. Thus, $\mathcal{S} \mathcal{F} \mathcal{S}$ consists of simplicial objects in the category of finite sets. Note that this is not the same as the category of finite simplicial sets since simplicial finite sets may have infinitely many nondegenerate simplices.

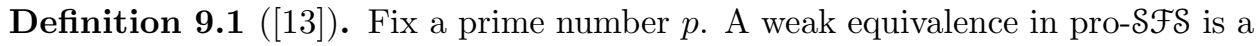
map $X \rightarrow Y$ such that $H^{*}(Y ; \mathbb{Z} / p) \rightarrow H^{*}(X ; \mathbb{Z} / p)$ is an isomorphism. A cofibration in pro-SFS is a map $X \rightarrow Y$ such that $\lim X \rightarrow \lim Y$ is a cofibration of spaces.

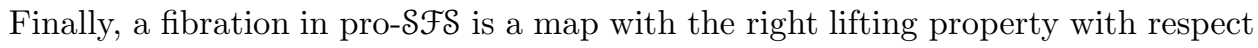
to trivial cofibrations.

Theorem 9.2 (13]). The above definitions give a model structure on the category pro-SFS.

Pro-finite completion gives an adjoint pair of functors between pro- $\mathcal{S} \mathcal{S}$ and pro-SFS. Sullivan [15] defined a topological pro-finite completion functor $\wedge: \mathcal{S} \mathcal{S} \rightarrow$ pro-SFS. It is the extension to simplicial objects of the functor from sets to profinite sets sending $S$ to the pro-system of finite quotients of $S$. Pro-finite completion naturally extends to a functor $\wedge:$ pro-SS $\rightarrow$ pro- $\mathcal{S} \mathcal{F} \mathcal{S}$.

The following lemma generalizes the usual adjointness property of pro-finite completion.

Lemma 9.3. Pro-finite completion $\wedge:$ pro-SS $\rightarrow$ pro-SFS is left adjoint to the inclusion functor $i:$ pro-SFS $\rightarrow$ pro-SS.

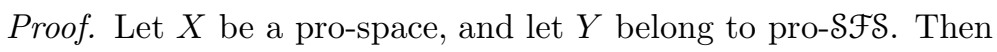

$$
\begin{aligned}
\operatorname{Hom}_{\text {pro-SFS }}\left(X^{\wedge}, Y\right) & =\lim _{s} \operatorname{colim}_{t} \operatorname{colim}_{u} \operatorname{Hom}_{\mathcal{S F S}}\left(\left(X_{t}^{\wedge}\right)_{u}, Y_{s}\right) \\
& =\lim _{s} \operatorname{colim}_{t} \operatorname{Hom}_{\mathcal{S} \mathcal{S}}\left(X_{t}, Y_{s}\right) \\
& =\operatorname{Hom}_{\text {pro-SS }}(X, i Y) .
\end{aligned}
$$

The second equality comes from the usual adjointness of pro-finite completion, which says that $\operatorname{colim}_{s} \operatorname{Hom}_{\mathcal{S} \mathcal{S}}\left(\left(Z^{\wedge}\right)_{s}, W\right)=\operatorname{Hom}_{\mathcal{S} \mathcal{S}}(Z, W)$ for any simplicial set $Z$ and any simplicial finite set $W$.

Proposition 9.4. The functors $\wedge$ and $i$ of Lemma 9.3 form a Quillen pair of adjoint functors. 
Proof. We must show that the left adjoint $\wedge$ preserves cofibrations and trivial cofibrations.

First, consider an inclusion $f: X \rightarrow Y$ of simplicial sets. The map $\lim _{s}\left(X^{\wedge}\right)_{s} \rightarrow$ $\lim _{t}\left(Y^{\wedge}\right)_{t}$ is an inclusion. This follows from the fact that if $x$ and $x^{\prime}$ are two distinct $n$-simplices of $\left(X^{\wedge}\right)_{s}$, then there is a finite quotient $\left(Y^{\wedge}\right)_{t}$ of $Y$ such that $f(x)$ and $f\left(x^{\prime}\right)$ are not equal.

Now consider an arbitrary cofibration of pro-spaces $f: X \rightarrow Y$. Without loss of generality, we may assume that $f$ is a levelwise cofibration. The $\operatorname{map} \lim _{t}\left(X_{s}^{\wedge}\right)_{t} \rightarrow$ $\lim _{t}\left(Y_{s}^{\wedge}\right)_{t}$ is an inclusion for each $s$, so the map

$$
\lim _{s} \lim _{t}\left(X_{s}^{\wedge}\right)_{t} \rightarrow \lim _{s} \lim _{t}\left(Y_{s}^{\wedge}\right)_{t}
$$

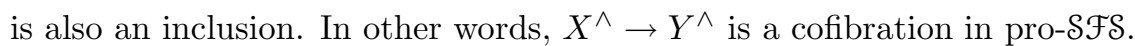

Next consider a trivial cofibration of pro-spaces $f: X \rightarrow Y$. We may assume that $f$ is a levelwise cofibration. Note that $H^{*}(Y ; \mathbb{Z} / p) \rightarrow H^{*}(X ; \mathbb{Z} / p)$ is an isomorphism by Theorem 7.3 .

Morel [13 1.2.2] observed that $H^{*}\left(X_{s} ; \mathbb{Z} / p\right) \cong H^{*}\left(X_{s}^{\wedge} ; \mathbb{Z} / p\right)$ since $\mathbb{Z} / p$ is a finite abelian group. Therefore

$$
\begin{aligned}
H^{*}\left(X^{\wedge} ; \mathbb{Z} / p\right) & =\underset{s}{\operatorname{colim}} \operatorname{colim}_{t} H^{*}\left(\left(X_{s}^{\wedge}\right)_{t} ; \mathbb{Z} / p\right) \\
& =\operatorname{colim}_{s} H^{*}\left(X_{s}^{\wedge} ; \mathbb{Z} / p\right) \\
& \cong \operatorname{colim}_{s} H^{*}\left(X_{s} ; \mathbb{Z} / p\right) \\
& =H^{*}(X ; \mathbb{Z} / p) .
\end{aligned}
$$

Similarly, $H^{*}\left(Y^{\wedge} ; \mathbb{Z} / p\right) \cong H^{*}(Y ; \mathbb{Z} / p)$. Hence $H^{*}\left(Y^{\wedge} ; \mathbb{Z} / p\right) \rightarrow H^{*}\left(X^{\wedge} ; \mathbb{Z} / p\right)$ is isomorphic to the map $H^{*}(Y ; \mathbb{Z} / p) \rightarrow H^{*}(X ; \mathbb{Z} / p)$, so it is also an isomorphism.

Corollary 9.5. The functors $\wedge$ and $i$ of Lemma 9.3 induce an adjoint pair on the homotopy categories $\mathrm{Ho}$ (pro-SS) and $\mathrm{Ho}($ pro-SFS).

\section{Strict Model Structure}

Edwards and Hastings [6, 3.3] defined a model structure on the category of prospaces and used it to study shape theory. We call this the "strict" structure because the weak equivalences are defined levelwise. We review their definitions and results.

Definition 10.1. A map of pro-spaces is a strict weak equivalence if it is isomorphic to a levelwise weak equivalence. A map of pro-spaces is a strict cofibration if it is a cofibration. A map of pro-spaces is a strict fibration if it has the right lifting property with respect to all strictly trivial cofibrations.

Definition 10.2. A map is a strong strict fibration if it is isomorphic to a level map of pro-spaces $X \rightarrow Y$ indexed by a cofinite directed set such that for all $t$,

$$
X_{t} \rightarrow Y_{t} \times \lim _{s<t} Y_{s} \lim _{s<t} X_{s}
$$

is a fibration.

Theorem $10.3([6])$. The above definitions give a model structure on the category pro-SS.

We call this the strict model structure on pro-SS. Note that Edwards and Hastings gave a more complicated definition for strict weak equivalences. The following proposition suffices to show that their definition and our definition coincide. 
Proposition 10.4. Strict weak equivalences are closed under composition.

Proof. Assume there are levelwise weak equivalences $f: X \rightarrow Y$ and $g: Z \rightarrow W$ with an isomorphism $h: Y \rightarrow Z$. We must construct a levelwise weak equivalence isomorphic to the composition $g h f$.

By adding isomorphisms to the systems for $f$ or $g$, make the cardinalities of the index sets equal. Choose an arbitrary isomorphism $\alpha$ from the index set of $g$ to the index set of $f$.

Define a function $t(s)$ inductively on height satisfying several conditions. Choose $t(s)$ large enough so that $Y_{t(s)} \rightarrow Z_{s}$ represents $h, t(s) \geq \alpha(s)$, and $t(u)<t(s)$ for all $u<s$.

The function $t$ defines cofinal subsystems of $X$ and $Y$. Hence we may assume that

$$
X \stackrel{f}{\longrightarrow} Y \stackrel{h}{\cong} Z \stackrel{g}{\longrightarrow} W
$$

is a level diagram indexed by a cofinite directed set $I$ where $f$ and $g$ are levelwise weak equivalences. However, the composition is not necessarily a levelwise weak equivalence because the map $h$ is not a levelwise weak equivalence.

Since $h$ is an isomorphism of pro-spaces, for every $s$, there exists $t>s$ and a commutative diagram

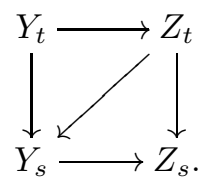

By restricting to cofinal subsystems, we may assume that such a diagram exists for every $t>s$.

Let $J$ be the directed set of indecomposable arrows of $I$. The domain and range functors $J \rightarrow I$ are both cofinal since $I$ is cofinite. For each $\phi: t \rightarrow s$ in $J$, factor the map $Z_{t} \rightarrow Y_{s}$ as

$$
Z_{t} \stackrel{i_{\phi}}{\longrightarrow} A_{\phi} \stackrel{p_{\phi}}{\longrightarrow} Y_{s}
$$

where $i_{\phi}$ is a cofibration and $p_{\phi}$ is a fibration.

Let $B_{\phi}$ be the pullback $X_{s} \times_{Y_{s}} A_{\phi}$, and let $C_{\phi}$ be the pushout $W_{t} \amalg_{Z_{t}} A_{\phi}$. These objects fit into a commutative diagram

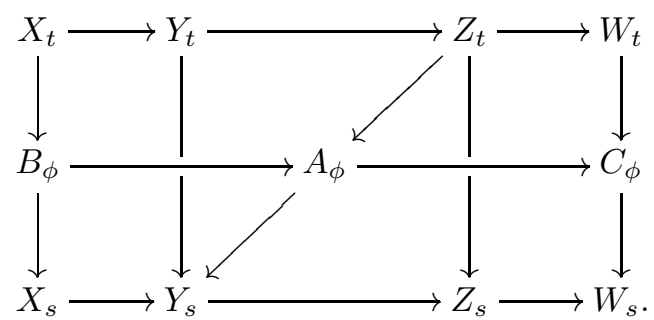

Note that $B_{\phi} \rightarrow A_{\phi}$ is a weak equivalence because it is a pullback of a weak equivalence along a fibration. Also, $A_{\phi} \rightarrow C_{\phi}$ is a weak equivalence because it is a pushout of a weak equivalence along a cofibration. Hence the composition $B \rightarrow A \rightarrow C$ is a levelwise weak equivalence. This composition is isomorphic to ghf since $B \cong X, Y \cong A \cong Z$, and $W \cong C$. 
The above proof works for any pro-category pro- $\mathcal{C}$ provided that $\mathcal{C}$ is a proper model category. In fact, a minor variation of the proof works when $\mathcal{C}$ is either left proper or right proper. It is possible to prove the other parts of the two-out-of-three axiom for strict weak equivalences with similar techniques.

Proposition 10.5 (6]). A map of pro-spaces is a strict fibration if and only if it is a retract of a strong strict fibration.

The relationship between the strict model structure and our model structure is expressed in the following results.

Let $\mathrm{Ho}_{\text {strict }}$ (pro-SS) be the homotopy category associated to the strict structure.

Proposition 10.6. The category $\mathrm{Ho}\left(\right.$ pro-SS) is a localization of $\mathrm{Ho}_{\text {strict }}$ (pro-SS).

Proof. Every levelwise weak equivalence is a weak equivalence in the sense of Definition 6.1

Corollary 10.7. If $f$ is a fibration, then $f$ is also a strict fibration.

Proof. The class of trivial cofibrations contains the class of strictly trivial cofibrations by Proposition 10.6.

Corollary 10.8. A map of pro-spaces is a trivial fibration if and only if it is a strictly trivial fibration.

Proof. Cofibrations are the same as strict cofibrations.

Proposition 10.9. Let $X$ be a pro-space, and let $Y$ be a pro-space such that each $Y_{s}$ has only finitely many non-zero homotopy groups. Then

$$
[X, Y]_{\text {pro }} \cong \operatorname{Hom}_{\mathrm{Ho}_{\text {strict }}(\text { pro-ss) }}(X, Y) \text {. }
$$

Proof. The condition on $Y$ ensures that its strictly fibrant replacement $Y^{\prime}$ is also a fibrant replacement. To calculate morphisms from $X$ to $Y$ in either homotopy category, consider morphisms from $X$ to $Y^{\prime}$ modulo the simplicial homotopy relation. Hence the morphisms are the same.

For example, the proposition applies when $Y$ is a system of Eilenberg-Mac Lane spaces. Edwards and Hastings described a relationship between homological algebra and the strict homotopy theory of such pro-spaces [6. Section 4]. It follows that the relationship works just as well for our homotopy theory of pro-spaces.

\section{Limits AND Colimits}

The rest of the paper concentrates on the technical details of the main theorems stated in Section 6 .

We provide specific constructions of limits and colimits in pro-categories. The existence of all colimits seems to be a little-known fact. In this section only, consider pro-objects indexed by arbitrary cofiltering categories, not just ones indexed by cofinite directed sets.

Proposition 11.1. If $\mathcal{C}$ is complete, then pro- $\mathcal{C}$ is also complete. If $\mathcal{C}$ is cocomplete, then pro- $\mathrm{C}$ is also cocomplete. 
Proof. Artin and Mazur 2, Appendix 4.2] showed that pro- $\mathcal{C}$ has all equalizers (resp. coequalizers) provided that $\mathcal{C}$ does. It suffices to construct arbitrary products (resp. coproducts) when these exist in $\mathcal{C}$.

Let $A$ be a set and let $X^{\alpha}$ be a pro-object for each $\alpha$ in $A$. Let $I_{\alpha}$ be the cofiltering index category for $X^{\alpha}$. Define $X=\prod_{\alpha \in A} X^{\alpha}$ to be the cofiltering system with objects $\prod_{\alpha \in B} X_{s_{\alpha}}^{\alpha}$ and index category $I$ consisting of pairs $\left(B,\left(s_{\alpha}\right)\right)$ where $B$ is a finite subset of $A$ and $\left(s_{\alpha}\right)$ is an element of $\prod_{\alpha \in B} I_{\alpha}$. A morphism of $I$ from $\left(B,\left(s_{\alpha}\right)\right)$ to $\left(C,\left(t_{\alpha}\right)\right)$ corresponds to an inclusion $C \subseteq B$ and morphisms $s_{\alpha} \rightarrow t_{\alpha}$ in $I_{\alpha}$ for all $\alpha$ in $C$. Use of finite subsets $B$ of $A$ is essential because we use the fact that finite products commute with filtered colimits.

Direct calculation shows that for any $Y$ in pro-e,

$$
\operatorname{Hom}_{\text {pro-e }}(Y, X) \cong \prod_{\alpha \in A} \operatorname{Hom}_{\text {pro-e }}\left(Y, X^{\alpha}\right) .
$$

Thus arbitrary products exist.

To construct the coproduct, define $X=\coprod_{\alpha \in A} X^{\alpha}$ to be the cofiltering system with objects $\coprod_{\alpha \in A} X_{s_{\alpha}}^{\alpha}$ and index category $\left\{\left(s_{\alpha}\right) \in \prod_{\alpha \in A} I_{\alpha}\right\}$. Note that $\prod I_{\alpha}$ is cofiltering since each $I_{\alpha}$ is.

In order to show that $X$ has the correct universal mapping property, it suffices to see that

$$
\operatorname{Hom}_{\text {pro-e }}(X, Y) \cong \prod_{\alpha \in A} \operatorname{Hom}_{\text {pro-e }}\left(X^{\alpha}, Y\right)
$$

for $Y$ any object of $\mathcal{C}$ (i.e., $Y$ is a constant system in pro- $\mathcal{C}$ ). This can be checked directly, using the fact that colimits indexed on product categories commute with the relevant products. Thus arbitrary coproducts exist.

\section{RetraCt Axioms}

The class of fibrations is obviously closed under retracts. The class of weak equivalences is closed under retracts because weak equivalences are defined in terms of isomorphisms of pro-local systems. We must show that the class of cofibrations is also closed under retracts. We prove a general result and then apply it to cofibrations.

Proposition 12.1. Let $\mathrm{C}$ be a category, and let $C$ be any class of maps in $\mathrm{C}$. Define the class $D$ as those maps in pro- $\mathrm{C}$ that are isomorphic to a level map that belongs to $C$ levelwise. Then $D$ is closed under retracts.

Proof. Suppose that $f: W \rightarrow Z$ is a retract of $g: X \rightarrow Y$, where $g$ is a level map that belongs to $C$ levelwise. Hence there is a commutative diagram

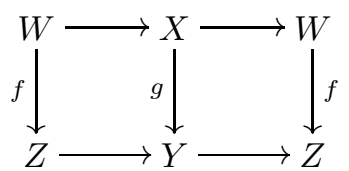

where the horizontal compositions are identity maps. We must show that $f$ also belongs to $D$. Choose a level representative for $f$.

By adding isomorphisms to the systems for $f$ or $g$, make the cardinalities of the index sets equal. Choose an arbitrary isomorphism $\alpha$ from the index set of $f$ to the index set of $g$. 
Define a function $t(s)$ inductively on height satisfying several conditions. First, choose $t(s)$ large enough so that $X_{t(s)} \rightarrow W_{s}$ and $Y_{t(s)} \rightarrow Z_{s}$ represent respectively the maps $X \rightarrow W$ and $Y \rightarrow Z$. Also, choose $t(s)$ large enough so that $t(s) \geq \alpha(s)$. Finally, choose $t(s)$ large enough so that for all $u<s, t(u)<t(s)$ with a commuting diagram

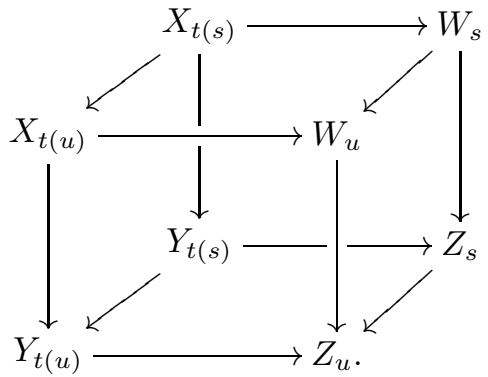

Now the function $t$ defines cofinal subsystems $\bar{X}$ and $\bar{Y}$ of $X$ and $Y$ where $\bar{X}$ and $\bar{Y}$ have the same index sets as $W$ and $Z$.

Repeat this process on $\bar{g}: \bar{X} \rightarrow \bar{Y}$ to obtain another function $u$ inducing cofinal subsystems $\bar{W}$ and $\bar{Z}$ of $W$ and $Z$. The result is a level diagram

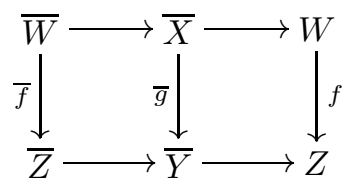

representing (up to isomorphism) $f$ as a retract of $g$.

Since $W \rightarrow X \rightarrow W$ and $Z \rightarrow Y \rightarrow Z$ are identity maps, $u$ can be chosen so that the composites $W_{u(s)}=\bar{W}_{s} \rightarrow \bar{X}_{s} \rightarrow W_{s}$ and $Z_{u(s)}=\bar{Z}_{s} \rightarrow \bar{Y}_{s} \rightarrow Z_{s}$ are structure maps of $W$ and $Z$ for all $s$.

Since $g$ belongs to $C$ levelwise, the same is true for $\bar{g}$. Define a pro-space $\hat{W}$ by starting with the system $W$ and replacing the single map $W_{u(s)} \rightarrow W_{s}$ with the pair of maps $W_{u(s)} \rightarrow \bar{X}_{s} \rightarrow W_{s}$. Define $\hat{Z}$ similarly.

Note that $W$ and $Z$ are cofinal subsystems of $\hat{W}$ and $\hat{Z}$ respectively, so $W$ is isomorphic to $\hat{W}$ and $Z$ is isomorphic to $\hat{Z}$. Thus it suffices to show that the level map $\hat{f}: \hat{W} \rightarrow \hat{Z}$ belongs to $D$.

The subsystem of $\hat{W}$ on objects $\left\{\bar{X}_{s}\right\}$ is also a cofinal subsystem, and the same is true for the subsystem on objects $\left\{\bar{Y}_{s}\right\}$ in $\hat{Z}$. Beware that the subsystem of $\hat{W}$ on $\left\{\bar{X}_{s}\right\}$ is not isomorphic to $\bar{X}$ because the structure maps are different. The same warning applies to $\left\{\bar{Y}_{s}\right\}$ and $\bar{Y}$.

Restrict $\hat{f}$ to the subsystem $\left\{\bar{X}_{s}\right\} \rightarrow\left\{\bar{Y}_{s}\right\}$. This last map belongs to $C$ levelwise, so it belongs to $D$. Hence $f$ also belongs to $D$ because $D$ is closed under isomorphisms.

Corollary 12.2. The class of cofibrations of pro-spaces is closed under retracts.

Proof. Apply Proposition 12.1 to the class of all cofibrations in $\mathcal{S} S$.

\section{WEAK EQUivalences}

We begin with the two-out-of-three axiom. 
Proposition 13.1. Let $f: X \rightarrow Y$ and $g: Y \rightarrow Z$ be maps of pro-spaces. If any two of the maps $f, g$, and $g f$ are weak equivalences, then so is the third.

Proof. For $n \geq 1$, the map $\Pi_{n} X \rightarrow f^{*} g^{*} \Pi_{n} Z$ factors as

$$
\Pi_{n} X \rightarrow f^{*} \Pi_{n} Y \rightarrow f^{*} g^{*} \Pi_{n} Z .
$$

Also, the map $\pi_{0} X \rightarrow \pi_{0} Z$ factors through $\pi_{0} Y$. This immediately proves two of the three cases.

For the third case, suppose that $f$ and $g f$ are weak equivalences. Then $f^{*} \Pi_{n} Y \rightarrow$ $f^{*} g^{*} \Pi_{n} Z$ is an isomorphism for all $n \geq 1$. By Lemma [5.2, $\Pi_{n} Y \rightarrow g^{*} \Pi_{n} Z$ is also an isomorphism for all $n \geq 1$.

The following lemma is a surprising generalization to pro-groups of an obvious fact about groups. Bousfield and Kan [4, III.2.2] stated without proof a special case.

Lemma 13.2. Let $U$ be the forgetful functor from pro-groups to pro-sets. Then a map $f$ of pro-groups is an isomorphism if and only if $U(f)$ is an isomorphism of pro-sets.

Proof. For simplicity write the group operations additively, even though the groups are not necessarily abelian.

We may assume that $f: X \rightarrow Y$ is a level map. If $f$ is an isomorphism, then $U f$ is also an isomorphism by Lemma 2.3.

Now suppose that $U f$ is an isomorphism. By Lemma 2.3 applied twice, for every $s$, there exist $u \geq t \geq s$ and a commutative diagram

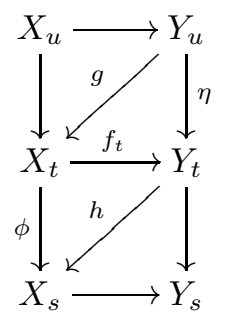

where the diagonal maps are not necessarily group homomorphisms.

However, the composite map $Y_{u} \rightarrow X_{s}$ is in fact a group homomorphism. For every $x$ and $y$ in $Y_{u}$,

$$
h \eta(x+y)=h(\eta x+\eta y)=h\left(f_{t} g x+f_{t} g y\right)=h f_{t}(g x+g y)
$$

because of commutativity in the top square. Also,

$$
h f_{t}(g x+g y)=\phi(g x+g y)=\phi g x+\phi g y
$$

because of commutativity in the bottom square. Finally, $\phi g=h \eta$.

Therefore, there is a commutative diagram of groups

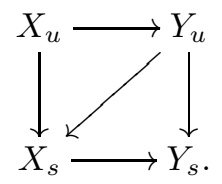

By Lemma 2.3 $f$ is an isomorphism. 
The formal nature of Definition 6.1 is often too abstract for comfort in technical situations. The following proposition shows that conditions ( $a$ ) and (b) of Theorem 7.3 are equivalent, thus giving a less natural but more concrete equivalent definition of weak equivalence.

Proposition 13.3. A level map of pro-spaces $f:(X, \phi) \rightarrow(Y, \eta)$ is a weak equivalence if and only if for all $n \geq 0$ and for all $s$, there exists some $t \geq s$ such that for all basepoints $*$ in $X_{t}$, there is a commutative diagram

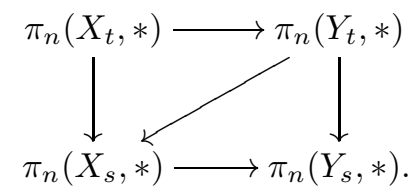

Remark 13.4. By the argument in the proof of Lemma 13.2, it is not important whether we assume that the diagonal map is a group homomorphism or just a map of sets. For convenience, we assume that it is a group homomorphism. Note that the diagonal map is not geometrically induced; it just exists abstractly. The choice of $t$ may depend on $n$ and $s$, but it must work for every basepoint.

Proof. First suppose that $f$ is a weak equivalence. Since $\pi_{0} f$ is an isomorphism, Lemma 2.3 gives the conclusion for $n=0$. For $n \geq 1$, Lemma 2.3 implies that, for every $s$, there exists a $t \geq s$ and a commutative diagram

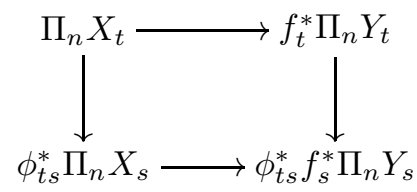

of local systems on $X_{t}$.

In particular, for every basepoint $*$ in $X_{t}$, there is a commutative diagram

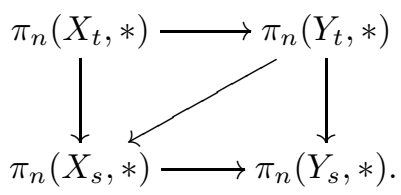

This proves the "only if" part of the claim.

Now suppose that the diagrams in the statement of the proposition exist. By Lemma 2.3. $\pi_{0} f$ is an isomorphism. For $n \geq 1$, we use the fact that a local system $L$ on a space $Z$ is determined up to isomorphism by its value $L_{x}$ as a $\pi_{1}(Z, x)$-module for one point $x$ in each component of $Z$. For every $s$, there exist $u \geq t \geq s$ such that for every basepoint $*$ in $X_{u}$ there are commutative diagrams
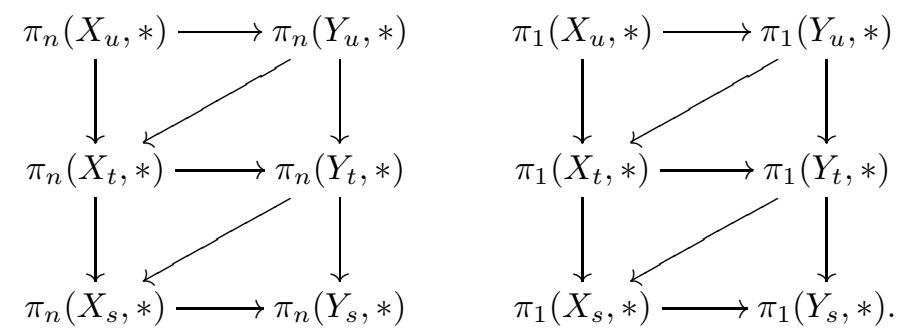
A diagram chase like that in the proof of Lemma 13.2 shows that the map $\pi_{n}\left(Y_{u}, *\right) \rightarrow \pi_{n}\left(X_{s}, *\right)$ is actually a map of $\pi_{1}\left(X_{u}, *\right)$-modules, even though the diagonal maps in the left diagram above are not maps of $\pi_{1}\left(X_{u}, *\right)$-modules. This defines a commutative diagram

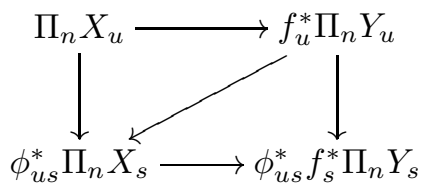

of local systems on $X_{u}$.

Hence $\Pi_{n} X \rightarrow f^{*} \Pi_{n} Y$ is an isomorphism by Lemma 2.3

Corollary 13.5. Suppose that $f: X \rightarrow Y$ is a level map of pro-spaces indexed by a cofinite directed set $I$ for which there is a strictly increasing function $n: I \rightarrow \mathbb{N}$ such that $f_{s}: X_{s} \rightarrow Y_{s}$ is an $n(s)$-equivalence. Then $f$ is a weak equivalence.

Proof. For any $s$ in $I$ and any $n \geq 0$, choose $t \geq s$ such that $n(t)>n$. For every point $*$ in $X_{t}$, there is a commutative diagram of solid arrows

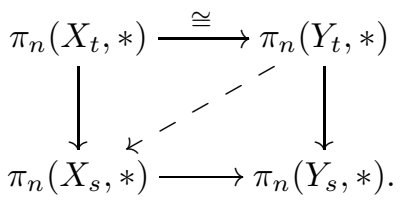

Since the top horizontal map is an isomorphism, this diagram can be extended to include the dashed arrow. Thus $f$ satisfies the condition of Proposition 13.3, so it is a weak equivalence.

\section{Fibrations}

The following lemma states some useful properties of strong fibrations that follow directly from the definition.

Lemma 14.1. If $f: X \rightarrow Y$ is a strong fibration, then for all the maps $f_{t}: X_{t} \rightarrow$ $Y_{t}$ and $g_{t}: \lim _{s<t} X_{s} \rightarrow \lim _{s<t} Y_{s}$ are co-n $(t)$-fibrations.

Proof. Let $h_{t}$ be the map $X_{t} \rightarrow Y_{t} \times_{\lim _{s<t} Y_{s}} \lim _{s<t} X_{s}$, so $h_{t}$ is a co- $n(t)$-fibration by the definition of strong fibrations.

We use the lifting property characterization of co- $n(t)$-fibrations. For the purposes of induction, assume that the maps $f_{s}$ and $g_{s}$ are co- $n(s)$-fibrations for all $s<t$. In particular, they are all co- $n(t)$-fibrations since $n(s) \leq n(t)$.

Let $A \rightarrow B$ be an $n(t)$-cofibration. A lift in the diagram

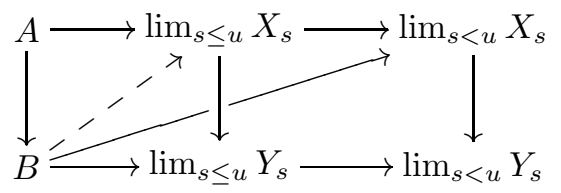


is the same as a lift for

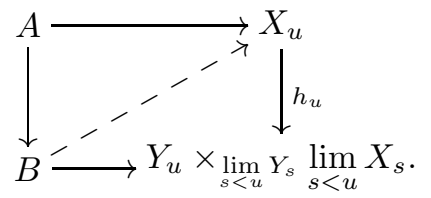

A lift exists in the last diagram because $h_{u}$ is a co- $n(u)$-fibration. Hence lifts can be extended inductively to obtain lifts for $g_{t}$. Thus $g_{t}$ is a co- $n(t)$-fibration.

The projection $p_{t}: Y_{t} \times \lim _{s<t} Y_{s} \lim _{s<t} X_{s} \rightarrow Y_{t}$ is the pullback of $g_{t}$ along the base $Y_{t} \rightarrow \lim _{s<t} Y_{s}$. Therefore, it is also a co- $n(t)$-fibration since right lifting properties are preserved by pullbacks. Now $f_{t}=p_{t} h_{t}$, so $f_{t}$ is a co- $n(t)$-fibration since it is a composition of such maps.

The following proposition is useful for recognizing that particular maps are strong fibrations.

Proposition 14.2. A level map $f: X \rightarrow Y$ is a strong fibration if and only if for all $t, f_{t}: X_{t} \rightarrow Y_{t}$ is a co- $n(t)$-equivalence and

$$
h_{t}: X_{t} \rightarrow Y_{t} \times \lim _{s<t} Y_{s} \lim _{s<t} X_{s}
$$

is a fibration.

Proof. One direction was proved in Lemma 14.1.

Use the notation of Lemma 14.1 Suppose that $f_{t}$ is a co- $n(t)$-equivalence and $h_{t}$ is a fibration for all $t$. It suffices to show that $h_{t}$ is also a co- $n(t)$-equivalence. For the purposes of induction, assume that $h_{s}$ is a co- $n(s)$-equivalence for $s<t$.

As in the proof of Lemma 14.1, $g_{t}$ is a co- $n(t)$-fibration by the inductive assumption. Hence $p_{t}$ also is a co- $n(t)$-fibration. The maps $p_{t}$ and $f_{t}$ are co- $n(t)$ equivalences. Since $f_{t}=p_{t} h_{t}$, it follows that $h_{t}$ is also a co- $n(t)$-equivalence.

Our next goal is to show that strong fibrations are also fibrations. This does not follow immediately from the definitions. First we need two preliminary lemmas about simplicial sets.

Lemma 14.3. Suppose given a commutative diagram of simplicial sets

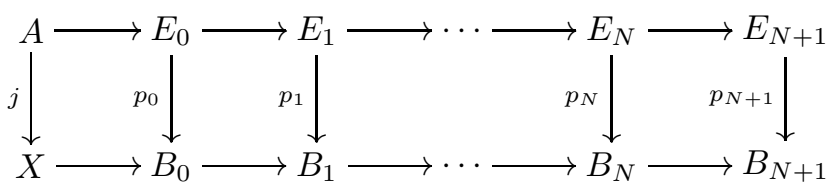

such that $j$ is a cofibration, every $p_{i}$ is a fibration, and $p_{N+1}$ is a co-N-fibration.

For every choice of basepoint $*$ in $E_{i}$, there is an induced map $F_{i} \rightarrow F_{i+1}$ where $F_{i}$ and $F_{i+1}$ are fibers of $p_{i}$ and $p_{i+1}$. Suppose that for every $i \leq N-1$ and every point $*$ in $E_{i}$, the map $\pi_{i}\left(F_{i}, *\right) \rightarrow \pi_{i}\left(F_{i+1}, *\right)$ is trivial. Finally, suppose that the image of $\pi_{0} E_{0}$ in $\pi_{0} B_{0}$ contains the image of $\pi_{0} X$. Then there is a lift in the 
diagram

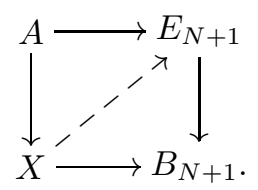

Proof. There exists a lift in the diagram

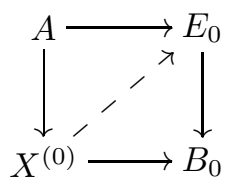

because the image of $\pi_{0} E_{0}$ in $\pi_{0} B_{0}$ contains the image of $\pi_{0} X$.

Now consider a diagram

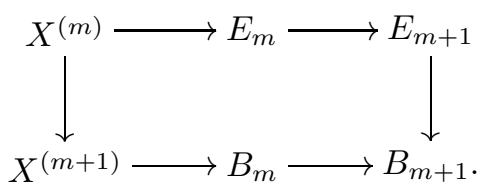

We have already shown that this diagram exists for $m=0$. We show by induction that this diagram exists for all $m \leq N$. The induction step amounts to finding a lift in the above diagram.

We construct a lift one simplex at a time. Therefore, it suffices to assume that $X^{(m)}=\partial \Delta^{m+1}$ and $X^{(m+1)}=\Delta^{m+1}$. The obstruction to finding a lift is an element of $\pi_{m} F_{m+1}$, and this obstruction lies in the image of $\pi_{m} F_{m}$. When $m<N$ the map $\pi_{m} F_{m} \rightarrow \pi_{m} F_{m+1}$ is trivial, so there is no obstruction to lifting.

By induction on $m$, there is a diagram

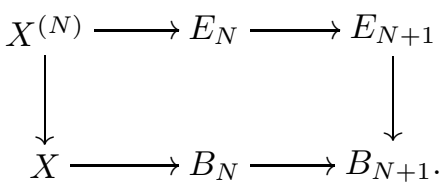

A lift exists in this diagram since the left vertical arrow is an $N$-cofibration and the right vertical arrow is a co- $N$-fibration. The map $X \rightarrow E_{N+1}$ is the desired lifting.

Lemma 14.4. Suppose that $n \geq 0$ and given a commutative diagram of simplicial sets

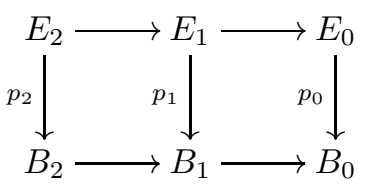

such that each $p_{i}$ is a fibration. For every choice of basepoint $*$ in $E_{2}$, there are induced maps $F_{2} \rightarrow F_{1} \rightarrow F_{0}$ where $F_{i}$ is the fiber of $p_{i}$ over the basepoint.

Suppose that for every $*$ in $E_{2}$, the maps $\pi_{n}\left(E_{2}, *\right) \rightarrow \pi_{n}\left(E_{1}, *\right)$ and $\pi_{n+1}\left(B_{1}, *\right)$ $\rightarrow \pi_{n+1}\left(B_{0}, *\right)$ factor through $\pi_{n}\left(B_{2}, *\right)$ and $\pi_{n+1}\left(E_{0}, *\right)$ respectively. Then for every $*$, the map $\pi_{n}\left(F_{2}, *\right) \rightarrow \pi_{n}\left(F_{0}, *\right)$ is trivial. 
Proof. Chase the commutative diagram with exact rows

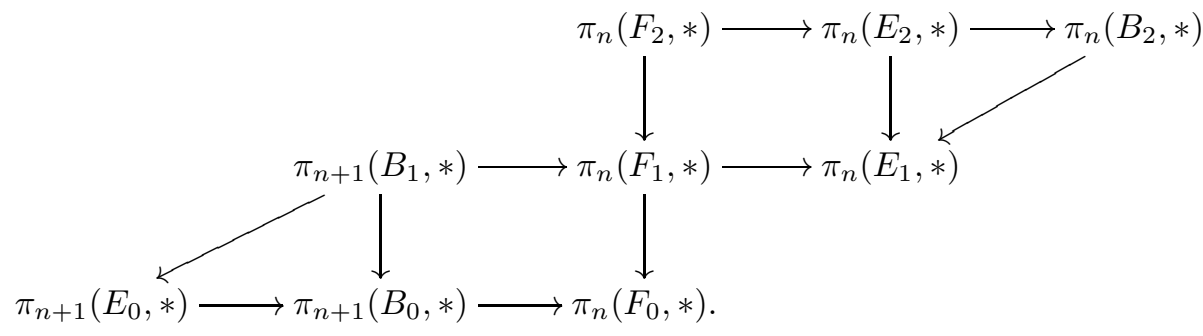

The next proposition shows that strong fibrations are also fibrations. This result is the technical heart of the whole theory.

Proposition 14.5. Strong fibrations have the right lifting property with respect to trivial cofibrations.

Proof. Consider a commutative diagram of pro-spaces

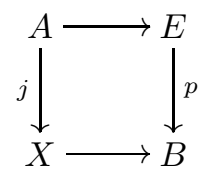

such that $j$ is a trivial cofibration and $p$ is a strong fibration. We may assume that $j$ is a level map that is a levelwise cofibration. By choosing an appropriate cofinal subsystem for $j$ as in the proof of Proposition 12.1, we may additionally assume that the square diagram is a level diagram. This choice preserves $j$ as a levelwise cofibration.

We construct a lifting by induction. Suppose that for all $s<t$, there exists $a(s) \geq s$ and a map $X_{a(s)} \rightarrow E_{s}$ such that for all $u<s<t, a(u) \leq a(s)$ and there is a commutative diagram

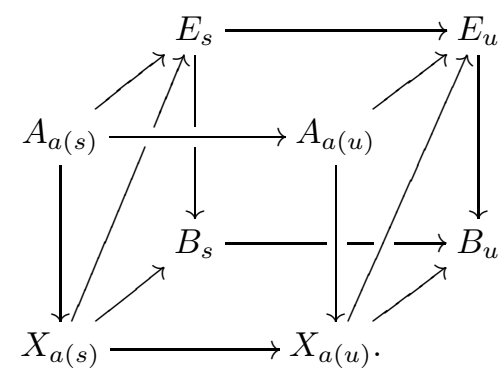

To extend the map $X \rightarrow E$ to level $t$, we must find a lift in the diagram

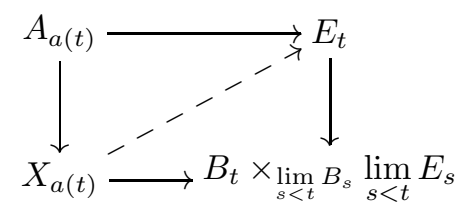

for some $a(t) \geq t$ such that $a(s)<a(t)$ for all $s<t$. 
Write the map $E_{t} \rightarrow B_{t} \times_{\lim _{s<t} B_{s}} \lim _{s<t} E_{s}$ as $q: W \rightarrow Z$. Because $p: E \rightarrow B$ is a strong fibration, $q$ is a co- $N$-fibration for some $N$.

Choose $t_{2 N}$ so that $t_{2 N}>a(s)$ for all $s<t$. Now select $t_{2 N-1}, t_{2 N-2}, \ldots, t_{1}, t_{0}$ so that $t_{i}>t_{i+1}$ and there exist commutative diagrams

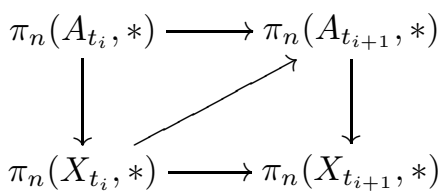

for all $0 \leq n \leq N$ and all basepoints $*$ in $A_{t_{i}}$. This is possible since $j$ is a weak equivalence and there are only finitely many conditions on the choice of each $t_{i}$.

Finally, choose $a(t)$ so that $a(t)>t_{0}$ and there exists a commutative diagram

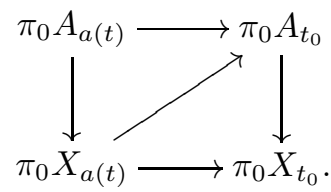

Functorially factor each map $A_{t_{i}} \rightarrow X_{t_{i}}$ as $A_{t_{i}} \stackrel{a_{i}}{\longrightarrow} Y_{t_{i}} \stackrel{b_{i}}{\longrightarrow} X_{t_{i}}$, where $a_{i}$ is a trivial cofibration and $b_{i}$ is a fibration.

Choose a basepoint $*$ in $Y_{t_{i}}$. Since $a_{i}$ is a weak equivalence, there exists a basepoint $\sharp$ in $A_{t_{i}}$ such that there is a path in $Y_{t_{i}}$ from $*$ to the image of $\sharp$. For $0 \leq n \leq N$, there is a diagram of solid arrows

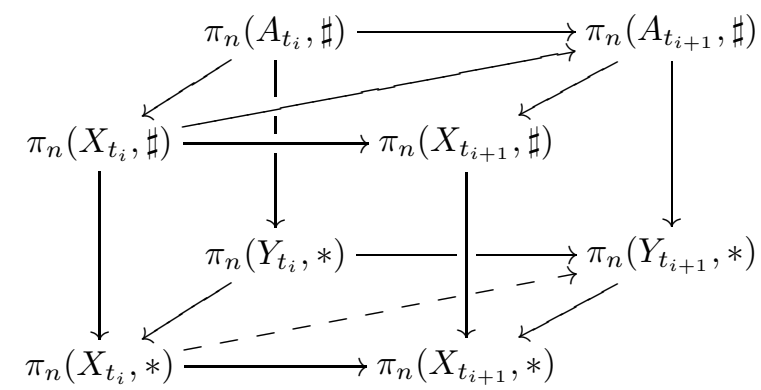

where the vertical arrows are induced by the choice of path. Since the vertical arrows are all isomorphisms, the dashed arrow also exists.

For similar reasons, there is also a commutative diagram

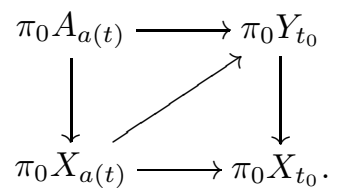


Now we have

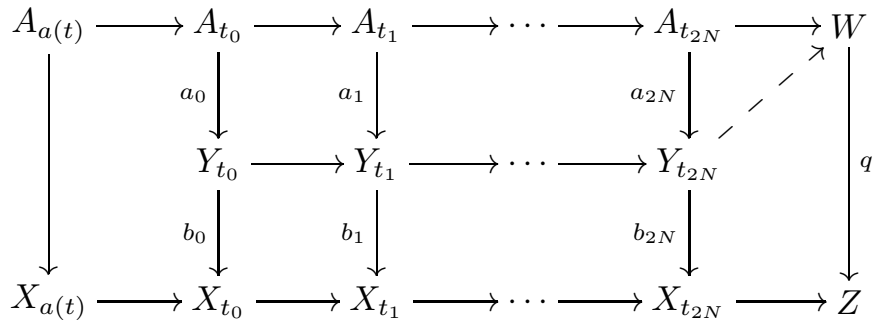

where the dashed arrow exists because $a_{2 N}$ is a trivial cofibration and $q$ is a fibration. So we need only find a lift for the diagram

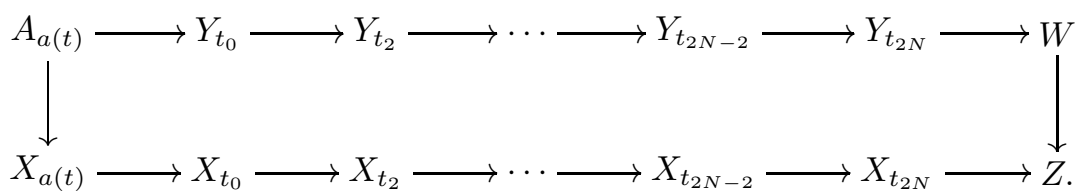

Lemma 14.4 tells us that this diagram satisfies the hypotheses of Lemma 14.3 Hence the desired lift exists.

\section{Lifting AND FACTORIZATION Axioms}

We now prove the lifting and factorizations axioms.

Proposition 15.1. If $f: X \rightarrow Y$ is a map of pro-spaces, then $f$ factors (not functorially) as

$$
X \stackrel{i}{\longrightarrow} Z \stackrel{p}{\longrightarrow} Y,
$$

where $i$ is a trivial cofibration and $p$ is a strong fibration.

Proof. We may assume that $f$ is a level map. We construct the factorization inductively.

Assume for the sake of induction that the factorization is already constructed on all indices less than $t$. Recall the height function $h(t)$ from Definition 2.2, Factor

$$
X_{t} \rightarrow Y_{t} \times \lim _{s<t} Y_{s} \lim _{s<t} Z_{s}
$$

as

$$
X_{t} \stackrel{i_{t}}{\longrightarrow} Z_{t} \stackrel{q_{t}}{\longrightarrow} Y_{t} \times \lim _{s<t} Y_{s} \lim _{s<t} Z_{s}
$$

where $i_{t}$ is an $h(t)$-cofibration and $q_{t}$ is a co- $h(t)$-fibration. Let $p_{t}: Z_{t} \rightarrow Y_{t}$ be the projection map induced by $q_{t}$. This extends the factorization to level $t$.

Now $p$ satisfies the definition of strong fibration by construction. Also, $i$ is a levelwise cofibration by construction. Finally, $i$ is a weak equivalence by Corollary 13.5.

Proposition 15.2. If $f: X \rightarrow Y$ is a map of pro-spaces, then $f$ factors (not functorially) as

$$
X \stackrel{i}{\longrightarrow} Z \stackrel{p}{\longrightarrow} Y
$$

where $i$ is a cofibration and $p$ is a trivial fibration. 
Proof. Use the strict model structure to factor $f$ as a cofibration $i$ followed by a strictly trivial fibration $p$. Then $p$ is also a trivial fibration by Corollary 10.8 .

Remark 15.3. We rely here on factorizations in the strict model structure. These factorizations are constructed similarly to the method of the proof of Proposition 15.1 .

One of the lifting axioms is automatic from the definition of fibrations. The other axiom is proved in the following proposition.

Proposition 15.4. Cofibrations have the left lifting property with respect to trivial fibrations.

Proof. Consider a commutative diagram of pro-spaces

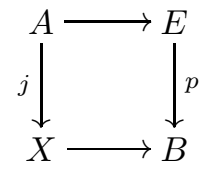

such that $j$ is a cofibration and $p$ is a trivial fibration. We may assume that $j$ is a level map that is a levelwise cofibration. By choosing an appropriate cofinal subsystem for $j$, we may additionally assume that the square diagram is a level diagram. This choice preserves $j$ as a levelwise cofibration.

Use the strict model structure to factor $p$ as $E \stackrel{i}{\longrightarrow} Y \stackrel{q}{\longrightarrow} B$, where $i$ is a levelwise cofibration and $q$ is a strictly trivial fibration. Note that $i$ is a weak equivalence by the two-out-of-three axiom as proved in Proposition 13.1.

In the diagram

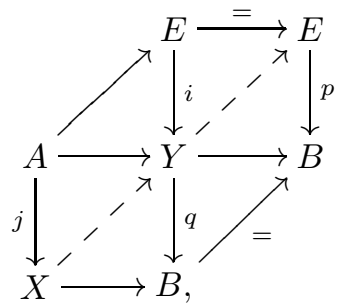

the lift in the lower left square exists because of the strict model structure, and the lift in the upper right square exists by the definition of fibrations. The composition $X \rightarrow Y \rightarrow E$ is the desired lift.

\section{Simplicial Model Structure}

Recall that a model structure on a category $\mathcal{C}$ is simplicial if for every $X$ in $\mathcal{C}$ and every simplicial set $K$, there are functorial constructions $X \otimes K$ ("tensor") and $X^{K}$ ("cotensor") in $\mathcal{C}$ satisfying certain associativity and unit conditions. Also, for every $X$ and $Y$ in $\mathcal{C}$, there is a simplicial function complex $\operatorname{Map}(X, Y)$. These three constructions are related by the adjunctions

$$
\operatorname{Map}(X \otimes K, Y) \cong \operatorname{Map}(K, \operatorname{Map}(X, Y)) \cong \operatorname{Map}\left(X, Y^{K}\right) .
$$

Finally, $\operatorname{Map}(-,-)$ must interact appropriately with the model structure as follows. If $i: A \rightarrow X$ is a cofibration in $\mathcal{C}$ and $p: E \rightarrow B$ is a fibration in $\mathcal{C}$, then

$$
\operatorname{Map}(X, E) \rightarrow \operatorname{Map}(A, E) \times_{\operatorname{Map}(A, B)} \operatorname{Map}(X, B)
$$


is a fibration that is a weak equivalence if either $i$ or $p$ is a weak equivalence.

We begin with a general proposition showing that tensors and cotensors defined for finite simplicial sets automatically extend to all simplicial sets.

Proposition 16.1. Suppose that $\mathcal{C}$ is a model category with a simplicial function complex $\operatorname{Map}(-,-)$. Also suppose that the tensor $X \otimes K$ and the cotensor $X^{K}$ are defined for all objects $X$ of $\mathcal{C}$ and all finite simplicial sets $K$ so that the axioms for a simplicial model structure are satisfied when they make sense. Then the definitions of tensor and cotensor can be extended to provide a simplicial model structure for e.

Proof. For any simplicial set $K$, let $K^{\text {fin }}$ be the filtering system of finite subspaces of $K$. For an object $X$ of $\mathcal{C}$, define $X \otimes K$ to be $\operatorname{colim}_{s}\left(X \otimes K_{s}^{\text {fin }}\right)$ and $X^{K}$ to be $\lim _{s} X^{K_{s}^{\mathrm{fin}}}$. Using the fact that the system $K^{\mathrm{fin}} \times L^{\mathrm{fin}}$ is cofinal in the system $(K \times L)^{\mathrm{fin}}$, the required isomorphisms

$$
X \otimes(K \times L) \cong(X \otimes K) \otimes L
$$

and

$$
\operatorname{Map}(X \otimes K, Y) \cong \operatorname{Map}(K, \operatorname{Map}(X, Y)) \cong \operatorname{Map}\left(X, Y^{K}\right)
$$

can be verified directly.

Definition 16.2. If $X$ and $Y$ are pro-spaces and $K$ is a simplicial set, define

$$
\begin{gathered}
\operatorname{Map}(X, Y)=\operatorname{Hom}_{\text {pro-ss }}\left(X \times \Delta^{\bullet}, Y\right)=\lim _{s} \operatorname{colim} \operatorname{Map}\left(X_{t}, Y_{s}\right), \\
X \otimes K=\underset{s}{\operatorname{colim}}\left(X \times K_{s}^{\mathrm{fin}}\right),
\end{gathered}
$$

and

$$
Y^{K}=\lim _{s}\left(Y^{K_{s}^{\mathrm{fin}}}\right) .
$$

For an arbitrary pro-space $X$ and a simplicial set $K, X \times K$ can be constructed as the levelwise product with $K$. Also, $\lim _{s} Y^{K_{s}^{\text {fin }}}$ can be constructed as the system $\left\{Y_{t}^{K_{s}^{\mathrm{fin}}}\right\}$, indexed by all pairs $(s, t)$ in the product of the index categories.

Note that $X \otimes K$ is not in general isomorphic to $X \times K$ because finite limits do not always commute with filtered colimits in the category of pro-spaces. However, if $K$ is finite, then $X \otimes K$ is isomorphic to $X \times K$ since $K$ itself is the terminal object of $K^{\text {fin }}$. Also, when $K$ is finite, $Y^{K}$ is the system $\left\{Y_{s}^{K}\right\}$ with the same index category as that of $Y$.

Proposition 16.3. The above definitions make pro-SS into a simplicial model category.

Proof. By Proposition 16.1, it suffices to check the axioms only for finite simplicial sets. Most of the axioms are obvious; we verify only the non-trivial ones here.

Let $X$ and $Y$ be arbitrary pro-spaces, and let $K$ be a finite simplicial set. We use the fact that $\operatorname{Hom}_{\mathcal{S} \mathcal{S}}\left(K, \operatorname{colim}_{s} Z_{s}\right)$ is equal to $\operatorname{colim}_{s} \operatorname{Hom}_{\mathcal{S} \mathcal{S}}\left(K, Z_{s}\right)$ for any filtered system $Z$ of simplicial sets because $K$ is finite. It follows by direct calculation that

$$
\operatorname{Map}(X \otimes K, Y) \cong \operatorname{Map}\left(X, Y^{K}\right) \cong \operatorname{Map}(K, \operatorname{Map}(X, Y)) .
$$

We now show that the map

$$
f: \operatorname{Map}(B, X) \rightarrow \operatorname{Map}(A, X) \times_{\operatorname{Map}(A, Y)} \operatorname{Map}(B, Y)
$$


is a fibration whenever $i: A \rightarrow B$ is a cofibration and $p: X \rightarrow Y$ is a fibration and that this map is a trivial fibration if either $i$ or $p$ is trivial. We proceed by showing that $f$ has the relevant right lifting property. Let $j: K \rightarrow L$ be a generating cofibration or a generating trivial cofibration. Note that $K$ and $L$ are finite simplicial sets.

By adjointness, it suffices to show that the map

$$
g: A \otimes L \amalg_{A \otimes K} B \otimes K \rightarrow B \otimes L
$$

is a cofibration that is trivial if either $i$ or $j$ is trivial.

We may assume that $i$ is a levelwise cofibration. For every $s, A_{s} \rightarrow B_{s}$ is a cofibration. Therefore, the map

$$
A_{s} \otimes L \amalg_{A_{s} \otimes K} B_{s} \otimes K \rightarrow B_{s} \otimes L
$$

is also a cofibration. This is a standard fact about simplicial sets. Thus $g$ is a levelwise cofibration.

In order to show that $g$ is trivial whenever $i$ or $j$ is, it suffices to show that the map $A \otimes K \rightarrow B \otimes K$ is trivial if $i$ is trivial and that the map $A \otimes K \rightarrow A \otimes L$ is trivial if $j$ is trivial. This reduction follows from the two-out-of-three axiom, the fact that trivial cofibrations are preserved by pushouts, and the commutative diagram

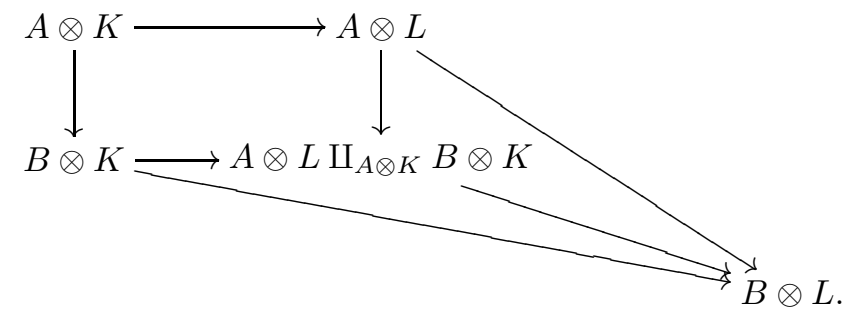

First suppose that $j$ is trivial. The map $A \otimes K \rightarrow A \otimes L$ is a levelwise weak equivalence, so it is a weak equivalence of pro-spaces.

Now suppose that $i$ is trivial. Since $A \otimes K \rightarrow B \otimes K$ is constructed by levelwise product with $K$, condition (b) of Theorem 7.3 is easily verified.

\section{Properness}

We now show that the model structure of Theorem 6.4 is proper. Recall that a model structure is left proper if weak equivalences are preserved under pushout along cofibrations. Dually, a model structure is right proper if weak equivalences are preserved under pullback along fibrations.

Proposition 17.1. The simplicial model structure of Theorem 6.4 is left and right proper.

Proof. Left properness follows immediately from the fact that all pro-spaces are cofibrant. We must show that the model structure is right proper.

Let $p: E \rightarrow B$ be a fibration and let $f: X \rightarrow B$ be a weak equivalence. Use Theorem 7.3 to suppose that $p$ and $f$ are level maps with the same cofinite directed index set $I$ for which there is a strictly increasing function $n: I \rightarrow \mathbb{N}$ such that $f_{s}$ is an $n(s)$-equivalence. Let $P$ be the pullback $X \times_{B} E$, which is constructed levelwise. We must show that the projection $P \rightarrow E$ is a weak equivalence. 
We start with a special case. First suppose that $p$ is a levelwise fibration. Let $*$ be a basepoint in $P_{s}$. This yields a diagram

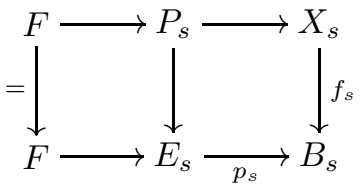

in which the rows are fiber sequences. From the 5-lemma applied to the long exact sequences of homotopy groups of the fibrations, $P_{s} \rightarrow E_{s}$ is also an $n(s)$-equivalence. By Theorem 7.3, $P \rightarrow E$ is a weak equivalence.

Now let $p$ be an arbitrary fibration. By Proposition 6.6, there exists a strong fibration $q: E^{\prime} \rightarrow B$ such that $p$ is a retract of $q$. Note that $q$ is a levelwise fibration by Lemma 14.1

Consider the commutative diagram

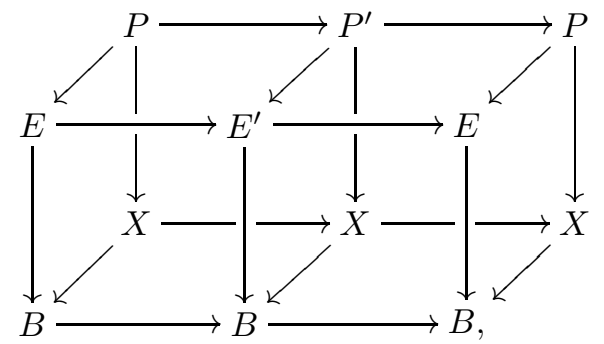

where $P^{\prime}=X \times_{B} E^{\prime}$. This diagram is a retract of squares in the sense that all of the horizontal compositions are identity maps. The map $P^{\prime} \rightarrow E^{\prime}$ is a weak equivalence by the special case. Since weak equivalences are closed under retracts, the map $P \rightarrow E$ is also a weak equivalence.

\section{Alternative Characterizations of Weak Equivalences}

We finish here the proof of Theorem 7.3 describing weak equivalences in other terms. For expository clarity, we split the theorem into several parts. The equivalence of $(a)$ and (b) was shown in Proposition 13.3 .

Proposition 18.1. A map of pro-spaces is a weak equivalence if and only if it is isomorphic to a level map $g: Z \rightarrow W$ indexed by a cofinite directed set $I$ for which there is a strictly increasing function $n: I \rightarrow \mathbb{N}$ such that $g_{s}: Z_{s} \rightarrow W_{s}$ is an $n(s)$-equivalence.

Proof. Corollary 13.5 showed that a map $g$ satisfying the conditions of the proposition is a weak equivalence.

Now suppose that $f$ is a weak equivalence. We may assume that $f$ is a level map. Use Proposition 15.1 to factor $f$ as

$$
X \stackrel{i}{\longrightarrow} Z \stackrel{p}{\longrightarrow} Y
$$

where $i$ is a trivial cofibration and $p$ is a trivial fibration. By Corollary 10.8, $p$ is also a strictly trivial fibration. In particular, $p$ is isomorphic to a levelwise weak equivalence. The proof of Proposition 15.1 indicates that $i$ satisfies the conditions of the proposition. By an argument similar to the proof of Proposition 10.4 $f$ also satisfies the conditions of the proposition. 
Recall the Moore-Postnikov functor $P$ from Definition [7.2,

Lemma 18.2. The canonical map $X \rightarrow P X$ is a weak equivalence for any prospace $X$.

Proof. Condition (b) of Theorem 7.3 is easily verified.

Proposition 18.3. A map of pro-spaces $f: X \rightarrow Y$ is a weak equivalence if and only if $P f$ is a strict weak equivalence.

Proof. Suppose that $P f$ is a strict weak equivalence. Then it is also a weak equivalence. The maps $X \rightarrow P X$ and $Y \rightarrow P Y$ are weak equivalences by Lemma 18.2 so $f$ is also.

Now suppose that $f$ is a weak equivalence. By Proposition 18.1, we may assume that $f$ is a level map indexed by a cofinite directed set $I$ for which there is a strictly increasing function $n: I \rightarrow \mathbb{N}$ such that $f_{s}: X_{s} \rightarrow Y_{s}$ is an $n(s)$-equivalence.

Consider the subsystem $X^{\prime}=\left\{P_{n(s)} X_{s} \mid s \in I\right\}$ of $X$ and the subsystem $Y^{\prime}=$ $\left\{P_{n(s)} Y_{s} \mid s \in I\right\}$ of $Y$. Note that $X^{\prime}$ and $Y^{\prime}$ are cofinal in $P X$ and $P Y$. Let $f^{\prime}$ be the level map $X^{\prime} \rightarrow Y^{\prime}$ induced by $P f$, so $f^{\prime}$ is isomorphic to $P f$. Since $X_{s} \rightarrow Y_{s}$ is an $n(s)$-equivalence, the map $P_{n(s)} X_{s} \rightarrow P_{n(s)} Y_{s}$ is a weak equivalence. Hence $f^{\prime}$ is a levelwise weak equivalence, so $P f$ is a strict weak equivalence.

Proposition 18.4. A map of pro-spaces $f: X \rightarrow Y$ is a weak equivalence if and only if $\pi_{0} f$ is an isomorphism of pro-sets, $\Pi_{1} X \rightarrow f^{*} \Pi_{1} Y$ is an isomorphism of pro-local systems on $X$, and for all $m$ and all local systems $L$ on $Y$, the map $H^{m}(Y ; L) \rightarrow H^{m}\left(X ; f^{*} L\right)$ is an isomorphism.

Proof. Let $f$ be a weak equivalence. By Proposition 18.1, we may assume that $f$ is a level map indexed by a cofinite directed set $I$ for which there is an increasing function $n: I \rightarrow \mathbb{N}$ such that $f_{s}$ is an $n(s)$-equivalence.

By the Whitehead theorem, $f_{s}$ induces a cohomology isomorphism in dimensions less than $n(s)$ for any local system on $Y_{s}$. Hence $f$ induces an isomorphism $H^{m}(Y ; L) \rightarrow H^{m}\left(X ; f^{*} L\right)$ in the colimit for every $m$.

Now suppose that $f$ satisfies the conditions of the proposition. Factor $f$ as

$$
X \stackrel{i}{\longrightarrow} Y^{\prime} \stackrel{p}{\longrightarrow} Y,
$$

where $i$ is a cofibration and $p$ is a strictly trivial fibration. Since $p$ induces cohomology isomorphisms by the first part of the proof, the map $i$ still satisfies the hypotheses of the proposition. Therefore, we may assume that $f$ is a level map that is a level cofibration.

Note that $M=\left(X \times \Delta^{1}\right) \amalg_{X} Y$ is weakly equivalent to $Y$ since $M$ is constructed levelwise and $M$ is levelwise weakly equivalent to $Y$.

We prove the proposition by showing that for every strongly fibrant pro-space $Z$, the map $\operatorname{Map}(M, Z) \rightarrow \operatorname{Map}(X, Z)$ is a weak equivalence. A retract argument then shows that the map $\operatorname{Map}(M, Z) \rightarrow \operatorname{Map}(X, Z)$ is a weak equivalence for all fibrant pro-spaces $Z$.

Assume that $Z$ is an arbitrary strongly fibrant pro-space. Note that each $Z_{s}$ is a fibrant simplicial set with only finitely many non-zero homotopy groups.

Recall that $\operatorname{Map}(X, Z)=\lim _{s} \operatorname{Map}\left(X, Z_{s}\right)$. Also recall that for every $t$, the map $Z_{t} \rightarrow \lim _{s \leq t} Z_{s}$ is a fibration since $Z$ is fibrant. Therefore, the map

$$
\operatorname{Map}\left(X, Z_{t}\right) \rightarrow \lim _{s \leq t} \operatorname{Map}\left(X, Z_{s}\right)=\operatorname{Map}\left(X, \lim _{s \leq t} Z_{s}\right)
$$


is a fibration. It follows that $\operatorname{Map}(X, Z)$ is weakly equivalent to the homotopy limit $\operatorname{holim}_{s} \operatorname{Map}\left(X, Z_{s}\right)$. Similarly, $\operatorname{Map}(M, Z)$ is weakly equivalent to the homotopy $\operatorname{limit}_{\operatorname{holim}} \operatorname{Map}\left(M, Z_{s}\right)$.

Since homotopy limits are invariant under levelwise weak equivalence, we only need show that $\operatorname{Map}\left(M, Z_{s}\right) \rightarrow \operatorname{Map}\left(X, Z_{s}\right)$ is a weak equivalence of simplicial sets for each $s$. Therefore, we may assume that $Z$ is a fibrant simplicial set with only finitely many non-zero homotopy groups.

By adjointness, to show that $\operatorname{Map}(M, Z) \rightarrow \operatorname{Map}(X, Z)$ is a weak equivalence, it suffices to find lifts in the diagrams of pro-spaces

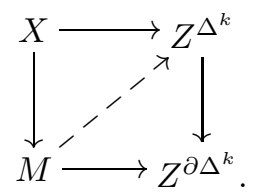

For simplicity, rewrite the fibration $Z^{\Delta^{k}} \rightarrow Z^{\partial \Delta^{k}}$ as $p: E \rightarrow B$. Our goal is to find an $s$ and a commuting diagram of simplicial sets

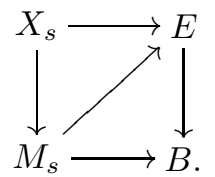

Note that $E$ and $B$ are fibrant simplicial sets, and $p$ is a fibration of simplicial sets. Use Moore-Postnikov systems [12, 8.9] to factor $E \rightarrow B$ as

$$
E \longrightarrow \cdots \longrightarrow E_{n} \stackrel{p_{n}}{\longrightarrow} E_{n-1} \longrightarrow \cdots \longrightarrow E_{2} \stackrel{p_{2}}{\longrightarrow} E_{1} \stackrel{p_{1}}{\longrightarrow} E_{0} \stackrel{p_{0}}{\longrightarrow} B
$$

where $E=\lim _{n} E_{n}$, each $p_{n}$ is a fibration, and the fibers of $p_{n}$ (which may vary up to homotopy because $E_{n-1}$ may not be connected) are of the form $K\left(\pi_{n} F, n\right)$ for some fiber $F$ of $p$.

The fibers of $p$ are of the form $\Omega^{k} Z$, so they have nonzero homotopy groups only in finitely many dimensions. Therefore, there exists $N$ such that $p_{n}$ is a weak equivalence for $n \geq N$.

We inductively construct partial liftings

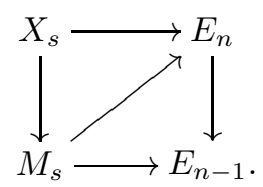

Since $E=\lim _{n} E_{n}$, these lifts assemble to give us the desired lifting.

Choose $t$ such that the original square of pro-spaces is represented by the square of simplicial sets

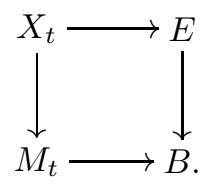


By the arguments of Lemma 14.3 and Proposition 14.5 there exists an $s_{0} \geq t$ and a commutative diagram

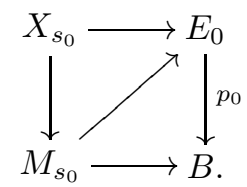

These arguments apply because $p_{0}$ is a co-1-fibration and because $X \rightarrow M$ induces an isomorphism in homotopy of dimension 0 and 1 .

For the moment, assume that $k \geq 1$. Since the fibers of $p$ are of the form $\Omega^{k} Z$, $\pi_{1} F$ is abelian for every fiber $F$ of $p$.

By obstruction theory (for example, [16]), the only obstruction to finding a lift

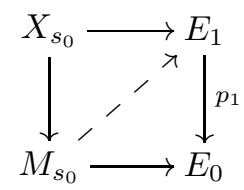

is a class $\alpha$ in $H^{2}\left(M_{s_{0}}, X_{s_{0}} ; L\right)$, where $L$ is the local system given by the first homotopy groups of the fibers of $p_{1}$. Note that $L$ makes sense because these homotopy groups are abelian.

But $H^{2}(M, X ; L)=0$ since $X \rightarrow M$ induces a cohomology isomorphism. Therefore, there exists an $s_{1} \geq s_{0}$ such that the image of $\alpha$ in $H^{2}\left(M_{s_{1}}, X_{s_{1}} ; L\right)$ is 0 . Hence, there is a lift in the above diagram when $s_{0}$ is replaced by $s_{1}$.

The same obstruction theory argument applies inductively to give liftings

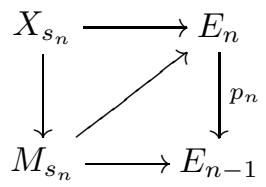

for each $2 \leq n \leq N$. Now $L$ is the local system given by $n$th homotopy groups of the fibers of $p_{n}$. Again, $L$ makes sense because $\pi_{1} F=0$ acts trivially on $\pi_{n} F$ for every fiber $F$ of $p_{n}$.

Let $s=s_{N}$. Recall that $p_{n}$ is a trivial fibration for $n \geq N$. Therefore, lifts exist inductively in the diagrams

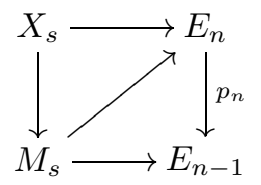

for $n>N$. Hence, the desired lifting exists when $k \geq 1$.

Now consider $k=0$. The argument given for $k \geq 1$ does not work. The trouble is that we cannot lift over $p_{1}$ with obstruction theory because the first homotopy groups of the fiber are not necessarily abelian.

When $k=0$, the map $p$ is just the map $Z \rightarrow *$, so we need to find an $s$ and a factorization of $X_{s} \rightarrow Z$ through $M_{s}$. Note that such factorizations are the same as factorizations up to homotopy of $X_{s} \rightarrow Z$ through $Y_{s}$.

Artin and Mazur [2, Section 4] constructed such factorizations when $Z$ is connected. Their argument works even when $Z$ is not connected, provided that 
$\pi_{0} X \cong \pi_{0} Y$. Here we use the fact that $[X, Z]_{\text {pro }}=\operatorname{Hom}_{\text {pro- } \mathrm{Ho}(\mathcal{S} s)}(X, Z)$ by Lemma 8.1 .

This proves the result.

\section{Non-Cofibrantly Generated Model Structures}

We prove in this section that the model structure of Section 6 is not cofibrantly generated. The same argument shows that the strict model structure 6 is also not cofibrantly generated. See Section 10 for a description of the strict structure. We start with a general lemma about cofibrantly generated model structures.

Lemma 19.1. Suppose that a model structure on a category $\mathcal{C}$ is cofibrantly generated with a set of generating cofibrations $I$. Let $T$ be the set of targets of maps in $I$, and let $X$ be any cofibrant object of $\mathcal{C}$ not isomorphic to the initial object. Then there exists some $Y$ in $T$ not isomorphic to the initial object with a map $Y \rightarrow X$ in $\mathrm{C}$.

Proof. Let $X$ be a cofibrant object of $\mathcal{C}$. Then $X$ is a retract of another object $X^{\prime}$, where $X^{\prime}$ is a transfinite composition of pushouts of maps in $I$ [10, 14.2.12]. Since there is a map from $X^{\prime}$ to $X$, it suffices to find a map from some object of $T$ to $X^{\prime}$. Since $X$ is not the initial object, $X^{\prime}$ is also not the initial object. Hence $X^{\prime}$ is a non-trivial transfinite composition of pushouts of maps in $I$. Let $Z \rightarrow Y$ be a map in $I$ occurring in the construction of $X^{\prime}$. Then there is a map from $Y$ to $X^{\prime}$.

The next proposition gives a construction of specific pro-sets with special properties.

Proposition 19.2. Let $F$ be a small family of pro-sets (i.e., a set of pro-sets) not containing the empty pro-set. Then there exists a pro-set $X$ such that for every $Y$ in $F$, there are no maps $Y \rightarrow X$ of pro-sets.

Proof. Choose an infinite cardinal $\kappa$ larger than the size of any of the sets occurring in any of the objects of $F$. Let $S$ be a set of size $\kappa$.

Define a pro-set $X$ as follows. Consider the collection of all subsets $U$ of $S$ whose complements $U^{c}$ are strictly smaller than $S$. Note that this implies that the size of $U$ is $\kappa$, but the converse is not true. These subsets form a pro-set, where the structure maps are inclusions. This system is cofiltered because $(U \cap V)^{c}=U^{c} \cup V^{c}$ is strictly smaller than $S$ when $U^{c}$ and $V^{c}$ are.

Let $Y$ be an object of $F$. Suppose that there is a map $f: Y \rightarrow X$ of pro-sets. Then there exists a $t$ and a map $f_{t, S}: Y_{t} \rightarrow S$ representing $f$. Let $A$ be the image of $f_{t, S}$, so $A$ is strictly smaller than $S$ since $Y_{t}$ is strictly smaller than $S$. Consider the set $S-A$, which occurs as an object in the system $X$. Since $f$ is a map of pro-sets, there exists a $u \geq t$ such that the composition $Y_{u} \rightarrow Y_{t} \rightarrow S$ factors through $S-A$. Since $Y_{t}$ and $S-A$ have disjoint images in $S$, this is only possible if $Y_{u}$ is the empty set. However, $Y_{u}$ cannot be the empty set because $Y$ is not the empty pro-set. By contradiction, the map $f$ cannot exist.

Corollary 19.3. There are no cofibrantly generated model structures on pro-spaces for which every object is cofibrant.

Proof. We argue by contradiction. Suppose that there exists a cofibrantly generated model structure for which every object is cofibrant. Let $I$ be the set of generating 
cofibrations, and let $T$ be the set of targets of maps in $I$. Apply $\pi_{0}$ to $T$ to obtain a small family of pro-sets $F$.

Let $X$ be the pro-set constructed in Proposition 19.2. We can think of $X$ as a pro-space by identifying a set with a simplical set of dimension zero. By Lemma 19.1, there exists a non-empty $Y$ in $T$ and a map $Y \rightarrow X$. This induces a map $\pi_{0} Y \rightarrow \pi_{0} X=X$. However, such a map cannot exist by Proposition 19.2 because $\pi_{0} Y$ belongs to $F$.

This corollary applies, in particular, to the model structure of Section 6 and to the strict model structure.

\section{REFERENCES}

[1] M. Artin, A. Grothendieck, and J. L. Verdier, Theorie des topos et cohomologie étale des schemas, Lecture Notes in Mathematics, vol. 269, Springer-Verlag, 1972. MR 50:7130

[2] M. Artin and B. Mazur, Etale homotopy, Lecture Notes in Mathematics, vol. 100, SpringerVerlag, 1969. MR 39:6883

[3] A. K. Bousfield and E. M. Friedlander, Homotopy theory of $\Gamma$-spaces, spectra, and bisimplicial sets, Geometric Applications of Homotopy Theory, vol. II (Proc. Conf., Evanston, IL, 1977), Lecture Notes in Mathematics, vol. 658, Springer Verlag, 1978, pp. 80-130. MR 80e:55021

[4] A. K. Bousfield and D. Kan, Homotopy limits, completions, and localizations, Lecture Notes in Mathematics, vol. 304, Springer Verlag, 1972. MR 51:1825

[5] W. G. Dwyer and E. M. Friedlander, Algebraic and étale K-theory, Trans. Amer. Math. Soc. 292 (1985), 247-280. MR 87h:18013

[6] D. A. Edwards and H. M. Hastings, Čech and Steenrod homotopy theories with applications to geometric topology, Lecture Notes in Mathematics, vol. 542, Springer-Verlag, 1976. MR 55:1347. Addendum to: Čech and Steenrod homotopy theories with applications to geometric topology, Colloq. Math. 41 (1979), 161-163. MR 80m:55020

[7] E. M. Friedlander, Etale K-theory I: Connections with étale cohomology and algebraic vector bundles, Invent. Math. 60 (1980), 105-134. MR 82e:14029

[8] — Etale homotopy of simplicial schemes, Annals of Mathematics Studies, vol. 104, Princeton University Press, 1982. MR 84h:55012

[9] J. Grossman, A homotopy theory of pro-spaces, Trans. Amer. Math. Soc. 201 (1975), 161176. MR 50:8511

[10] P. Hirschhorn, Localization of Model Categories, preprint.

[11] M. A. Mandell, $E_{\infty}$ algebras and p-adic homotopy theory, Topology (to appear).

[12] J. P. May, Simplicial objects in algebraic topology, Van Nostrand Mathematical Studies, vol. 11, Van Nostrand, 1967. MR 36:5942

[13] F. Morel, Ensembles profinis simpliciaux et interprétation géométrique du foncteur T, Bull. Soc. Math. France 124 (1996), 347-373. MR 97k:55014

[14] D. G. Quillen, Homotopical algebra, Lecture Notes in Mathematics, vol. 43, Springer-Verlag, 1967. MR 36:6480

[15] D. Sullivan, Genetics of homotopy theory and the Adams conjecture, Ann. of Math. 100 (1974), 1-79. MR 56:1305

[16] G. W. Whitehead, Elements of homotopy theory, Graduate Texts in Mathematics, vol. 61, Springer Verlag, 1978. MR 80b:55001

Fakultät für Mathematik, Universität Bielefeld, 33501 Bielefeld, Germany

Current address: Department of Mathematics, University of Notre Dame, Notre Dame, Indiana 46556

E-mail address: isaksen.1@nd.edu 